\title{
Conditional resonance-fluorescence spectra of single atoms
}

\author{
Gerhard C. Hegerfeldt ${ }^{*}$ and Martin B. Plenio ${ }^{\dagger}$ \\ Institut für Theoretische Physik, Universität Göttingen, Bunsenstrasse 9, D-37073 Göttingen, Germany
}

(Received 28 March 1995; revised manuscript received 26 May 1995)

\begin{abstract}
The temporal behavior of a single quantum system may contain information, for instance, about macroscopic dark periods in electron shelving, which is washed out or even lost in ensemble averages. The same is true in principle for spectral information. For example, for an atom with light and dark periods one might try to spectrally analyze the light emitted in a light period of given length or, more generally, the light emitted into one solid angle while monitoring the temporal emission in another solid angle. Considering different emission behaviors one may get different spectra, i.e., spectra that are dependent on the conditions imposed. In this paper, such questions, in particular the notion of spectrum in a light period, are studied. To this end we generalize the recent quantum jump approach to include resonance-fluorescence spectra of single atoms. We explicitly show that the theory developed in this paper easily generalizes, e.g., to absorption spectra. We apply the theory to a $V$ system that has one metastable level and that may exhibit intermittent fluorescence when driven by two lasers. We show that the spectrum of resonance fluorescence of the complete ensemble differs substantially from that observed in a light period. A physical explanation of this behavior is presented.
\end{abstract}

PACS number(s): 42.50.Lc, 42.50.Ct, 32.90.+a

\section{INTRODUCTION}

Resonance fluorescence of laser-driven atomic multilevel systems and both its spectral as well as statistical, timeresolved, properties have been intensely studied. A famous example is the well-known Mollow spectrum $[1,2]$ of a laserdriven two-level system with a central peak and two sidebands for sufficiently strong driving. Other examples are the antibunching of light emitted by a single atom [3-5] and electron shelving or macroscopic dark periods [6,7]. Spectral properties usually involve ensembles and intensity correlations single systems [8].

More recently, we have investigated the stationary resonance-fluorescence spectrum of an ensemble of $V$ systems (cf. Fig. 1), which can exhibit extended light and dark periods, and it was shown analytically and numerically that there is an additional narrow peak on the strong transition [9]. We explained this as a consequence of the stochastic modulation of the resonance fluorescence by the intermittent dark periods. If correct this interpretation would imply that this narrow peak should be absent when the spectrum is observed when no dark periods occur. It therefore suggests itself to investigate the spectrum of a single atom in a sufficiently long light period to check this.

For this both spectral as well as temporal properties of the emitted radiation are required, and thus care is needed with the time-energy uncertainty relation. This can be done by assuming time-resolved photon measurements with a broadband detector in a solid angle $\Omega_{B}$ for the determination of light periods and by analyzing the spectrum in another, disjoint, solid angle, $\Omega_{S}$ say, as in Fig. 2 . The broadband detector in $\Omega_{B}$ is thus used as a device to trigger the spectrometer

\footnotetext{
*Electronic address: hegerf@theo-phys.gwdg.de

†Present address: The Blackett Laboratory, Imperial College, London SW7 2BZ, United Kingdom. Electronic address: m.plenio@ic.ac.uk
}

and to keep only spectral information conditioned to light periods of given length in the solid angle $\Omega_{B}$. More generally, one may consider a particular sequence of photon detection times, $t_{1}, t_{2}, \ldots$, in the broadband detector and only spectrally analyze the radiation in the solid angle $\Omega_{S}$ if this particular sequence occurs, or one can look for light periods of length at least $T$ and analyze the corresponding spectra at time $T$ after the beginning of the periods.

In this paper we will calculate such spectra for a single atom. To do this we give the correct generalization of the quantum jump approach (or Monte Carlo wave-function approach or quantum trajectories) $[10,11]$ applicable to our problem. The point is that one has to find the correct time evolution between successive detections in the broadband detector as well as the correct state after the detection of a photon. Since it is known that different choices can lead to the same results for the complete ensemble they need not describe a single system whose resonance fluorescence is

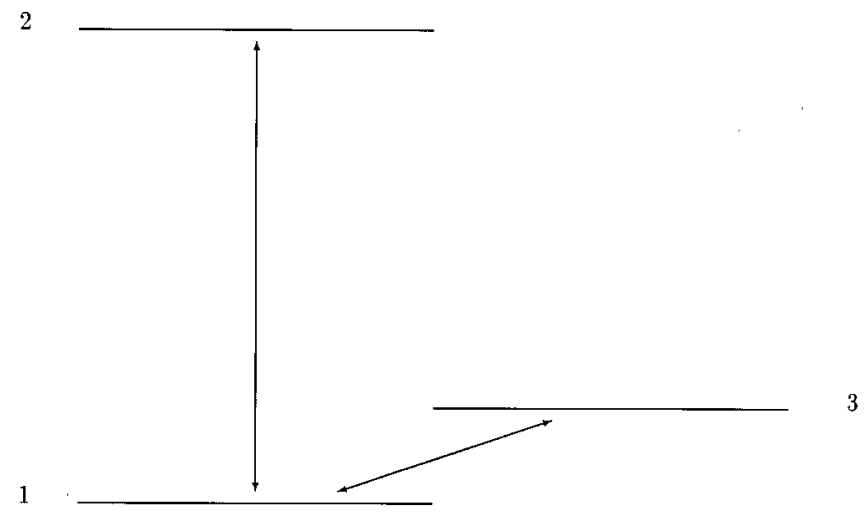

FIG. 1. The $V$ system. Two upper levels 2 and 3 couple to a common ground state 1 . The transition frequencies are assumed to be far apart so that each of the two lasers driving the system couples to only one of the transitions. The $2 \leftrightarrow 1$ transition is assumed to be strong while the $3 \leftrightarrow 1$ transition is weak. 


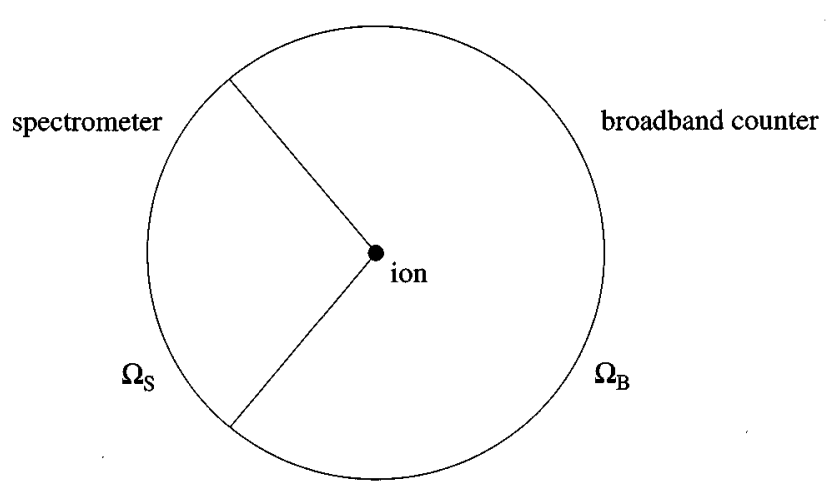

FIG. 2. Schematic picture of a possible experimental setup. The atom at the origin emits photons in all directions. In the solid angle $\Omega_{B}$ a broadband detector performs time-resolved measurements on the photons. A spectrometer is placed in the solid angle $\Omega_{S}$.

observed by a broadband photon counter. In fact, different possible evolution equations belong to different detection schemes, e.g., quantum diffusion equations to homodyne or heterodyne detection instead of photon counting. We first derive expressions for the expectation of conditional spectra, under the condition of given detection times in the broadband detector in the solid angle $\Omega_{B}$ and then average by a simulation procedure over many sequences of detection times compatible with the given light period.

For resonance-fluorescence spectra of the complete ensemble simulation approaches have already been discussed by other authors $[12,13]$. But questions concerning single systems and conditional spectra have not been treated and it is not obvious whether or not the results of Refs. $[12,13]$ can be used for such questions [14]. The results for the emission spectrum in Ref. [12] turn out to be a special case of our general equations in Sec. II below, although our methods are completely different.

The approach presented in this paper gives the evolution equations necessary for the calculation of conditional resonance-fluorescence spectra. It is based on physically observable quantities (photon numbers) and is easily generalized to other spectra, such as the absorption spectrum of a single atom, and it can also be used for efficient wavefunction simulations of these quantities for the complete ensemble. This may be interesting for the application to complex systems with a large number of energy levels.

Our paper is organized as follows. In Sec. II we generalize the quantum jump approach and develop a theory for the calculation of conditional resonance-fluorescence spectra. Generalizations to other quantities, such as, for example, the absorption spectra, are given. In Sec. III the theory is used to investigate the spectrum of resonance fluorescence of a single $V$ system in a light period. We find that such a spectrum can differ substantially from that of the complete ensemble. In particular, the narrow peak which we recently found in the spectrum of resonance fluorescence of the complete ensemble of $V$ systems [9] can vanish. We show that the reason is not a spectral broadening due to the finite length of the light periods - for this broadening can be made negligible by considering sufficiently long light periods - but rather to the fact that the resonance fluorescence in light periods is not modulated anymore by intermittent dark peri- ods. In Sec. IV we summarize and discuss the results.

\section{GENERAL THEORY}

Before generalizing it we recall that the usual quantum jump approach can be derived by considering rapidly repeated measurements of the complete quantized radiation field [15], where a detected photon is assumed to be absorbed, although this last assumption is not necessary [16]. The time evolution can then be divided into the evolution between successive detections and the determination of the state after a detection, the so-called reset state [11]. Let the measurements of the complete quantized radiation field ( $4 \pi$-broadband detector) be performed in rapid succession at times $s_{1}, \ldots, s_{n}$. The state at time $t=s_{n}$ of the subensemble for which in all these measurements no photon has been found is denoted by $\rho\left(s_{n} \mid \varnothing\right)$, where $\varnothing$ stands for empty set, and in a suitable interaction picture it is given in terms of the projection operator $\mathrm{P}_{0}$ onto the vacuum state of the quantized radiation field as

$$
\begin{aligned}
\rho\left(s_{n} \mid \varnothing\right)= & \mathbb{P}_{0} U_{I}\left(s_{n}, s_{n-1}\right) \mathbb{P}_{0} \cdots \mathbb{P}_{0} U_{I}\left(s_{1}, 0\right) \rho(0) \\
& \times U_{I}^{\dagger}\left(s_{1}, 0\right) \mathbb{P}_{0} \cdots \mathbb{P}_{0} U_{I}^{\dagger}\left(s_{n}, s_{n-1}\right) \mathbb{P}_{0},
\end{aligned}
$$

according to the von Neumann-Lüders projection postulate [17]. The normalization is chosen such that the trace of $\rho\left(s_{n} \mid \varnothing\right)$ is just the relative size of this subensemble, i.e., the probability for this event. This probability, and thus the trace, decreases with successive measurements since those systems which have emitted a photon leave the subensemble. The time difference $s_{k}-s_{k-1}$ of two successive measurements has to be small compared to all time constants of the atomic time evolution, to ensure that at most one photon is emitted in the time interval $\left[s_{k-1}, s_{k}\right]$. If the initial state is of the form

$$
\rho(0)=\rho_{A} \otimes\left|0_{\gamma}\right\rangle\left\langle 0_{\gamma}\right|,
$$

i.e., the quantized radiation field is initially in the vacuum state, one can now calculate $\mathrm{P}_{0} U\left(s_{k}, s_{k-1}\right) \mathrm{P}_{0}$ in second order perturbation theory. Setting $t=s_{n}$ one finds an expression of the form [15]

$$
\rho(t \mid \varnothing)=U_{r e d}(t, 0) \rho(0) U_{r e d}^{\dagger}(t, 0) .
$$

In a coarse-grained time scale this can be expressed as

$$
\dot{\rho}(t \mid \varnothing)=-\frac{i}{\hbar}\left\{H_{r e d} \rho(t \mid \varnothing)-\rho(t \mid \varnothing) H_{r e d}^{\dagger}\right\} .
$$

This presupposes that the time evolution $U_{\text {red }}(t, 0)$ maps pure states onto pure states [18]. With the chosen normalization of $\rho(t \mid \varnothing)$ the probability to find no photon in $[0, t]$ for a single atom is given by

$$
P_{0}(t \mid \rho(0))=\operatorname{tr}\{\rho(t \mid \varnothing)\},
$$

where $\rho(0)$ is the initial state at $t=0$.

If a photon is detected in the measurement at time $s_{n}$, but not before, the reset state after this detection is given by [11] 


$$
\begin{aligned}
\rho_{r}\left(s_{n}\right)= & \operatorname{tr}_{R}\left\{\left(\mathbb{1}-\mathrm{P}_{0}\right) U_{I}\left(s_{n}, s_{n-1}\right) \rho\left(s_{n-1} \mid \varnothing\right) U_{I}^{\dagger}\left(s_{n}, s_{n-1}\right)\right. \\
& \left.\times\left(\mathbb{1}-\mathrm{P}_{0}\right)\right\} \otimes \mathbb{P}_{0}
\end{aligned}
$$

where again the normalization is such that the trace of $\rho_{r}\left(s_{n}\right)$ is the probability for this event. It is assumed here that the detector absorbs the photon during the measurement process. Then the state after the detection is of the form (2) and one proceeds with Eq. (3) to continue the time evolution until the next detection of a photon. Equation (6) can be calculated in first order perturbation theory and one obtains an expression of the form [11]

$$
\rho_{r}(t)=\mathscr{R} \rho\left(s_{n-1} \mid \varnothing\right),
$$

where $\mathscr{B}$ is a suitable superoperator. If $\mathscr{B}$ maps pure states onto pure states one can find an operator $C$ such that

$$
\rho_{r}(t)=C \rho(t \mid \varnothing) C^{\dagger} .
$$

With the help of (3) and (7) or (4) and (8) one can now calculate the state of the atom for a given set of detection times, $t_{1}, t_{2}, \ldots$, say. On the other hand, one can also simulate the emission path of a single radiating atom with the help of the distribution of emission times derived from $P_{0}(t)$. If one would try to measure the spectrum of these photons one would find it extremely broadened, due to the time-energy uncertainty relation.

\section{A. Generalization of the quantum jump approach}

We now restrict the time-resolved broadband measurements to some solid angle $\Omega_{B}$, as in Fig. 2. If a photon is found, and absorbed, one has to project onto the vacuum state for all modes whose $\mathbf{k}$ vector points into the solid angle $\Omega_{B}$; to all other modes one has to apply the identity operator. Hence one cannot assume that the complete quantized radiation field is in the vacuum state as in the usual quantum jump approach. For the conditional spectrum in $\Omega_{S}$ we choose the photon number of particular modes as observables [14] and consider the subensemble where photons are found in the broadband detector at times $t_{1}, \ldots, t_{n}<t$ and no photons in all intervening measurements at times $s_{i}^{k}$ $\in\left[t_{k}, t_{k+1}\right], i=1, \ldots, m_{k}$, with $s_{0}^{k} \equiv t_{k}$. As in the usual quantum jump approach we assume that the $s_{j+1}^{k}-s_{j}^{k}$ are smaller than all the time constants of the atomic time evolution, but larger than the inverse transition frequencies. The density operator for this subensemble, in a suitable interaction picture, is denoted by $\rho\left(t \mid t_{1}, t_{2}, \ldots\right)$, where again the normalization is such that the trace is the probability of this event.

We now define the conditional quantity

$$
S_{\text {cond }}(\omega, \hat{\mathbf{k}} \lambda, t) \equiv \hbar \omega_{\mathbf{k} \lambda} \operatorname{tr}\left\{a_{\mathbf{k} \lambda}^{\dagger}(0) a_{\mathbf{k} \lambda}(0) \rho\left(t \mid t_{1}, t_{2}, \ldots\right)\right\},
$$

where $\omega=|\mathbf{k}| / c, \hat{\mathbf{k}}=\mathbf{k} /|\mathbf{k}|$, and we assume that $\mathbf{k}$ is a vector pointing into the solid angle $\Omega_{S}$ of the spectrometer. The conditional spectrum of resonance fluorescence is now obtained by summing over all vectors $\hat{\mathbf{k}}$ in $\Omega_{S}$. For the calculation of (9) we need the state $\rho\left(t \mid t_{1}, t_{2}, \ldots\right)$. With $\mathrm{P}_{0 \Omega_{B}}$ the projection operator onto the vacuum state for all modes with $\hat{\mathbf{k}} \in \Omega_{B}$ and with the abbreviation

$$
\mathscr{b} \equiv \mathrm{P}_{0 \Omega_{B}} U\left(t, s_{m_{n}}^{n}\right) \prod_{k=1}^{m_{n}} \mathrm{P}_{0 \Omega_{B}} U\left(s_{k}^{n}, s_{k-1}^{n}\right)
$$

we find, for $t_{n}<t<t_{n+1}$,

$$
\rho\left(t \mid t_{1}, t_{2}, \ldots\right)=\mathscr{C} \rho\left(t_{n}+0 \mid t_{1}, \ldots, t_{n-1}\right) \mathscr{b}^{\dagger},
$$

where $\rho\left(t_{n}+0 \mid t_{1}, \ldots, t_{n-1}\right)$ is the state right after the detection of a photon in $\Omega_{B}$; it is recursively given by

$$
\begin{aligned}
\rho\left(t_{n}+\right. & \left.0 \mid t_{1}, \ldots, t_{n-1}\right) \\
\equiv & \operatorname{tr}_{\Omega_{B}}\left\{\left(1-\mathbb{P}_{0 \Omega_{B}}\right) U\left(t_{n}, s_{m_{n-1}}^{n-1}\right)\right. \\
& \left.\times \rho\left(s_{m_{n-1}}^{n-1} \mid t_{1}, \ldots, t_{n-1}\right) U^{\dagger}\left(t_{n}, s_{m_{n-1}}^{n-1}\right)\left(1-\mathbb{P}_{0 \Omega_{B}}\right)\right\} \\
& \otimes \mathbb{P}_{0 \Omega_{B}} .
\end{aligned}
$$

Here $\operatorname{tr}_{\Omega_{B}}\{\}$ denotes the partial trace over all modes with a $\mathbf{k}$ vector that points into the solid angle $\Omega_{B}$. As in the usual quantum jump approach, at this point the assumption enters that the photons detected in the broadband detector are absorbed during the measurement as in a real counter. One can show, however, that this assumption is not necessary for obtaining the equations of motion of this section [16].

In the following we are going to derive the equations of motion for the quantity (9) and, more generally, for

$$
\operatorname{tr}\left\{a_{\mathbf{k} \lambda}^{\dagger} \boldsymbol{\sigma} a_{\mathbf{k} \lambda} \rho\left(t \mid t_{1}, t_{2}, \ldots\right)\right\},
$$

where

$$
\boldsymbol{\sigma}=\left(\sigma_{11}, \sigma_{22}, \sigma_{33}, \sigma_{21}, \sigma_{32}, \sigma_{13}, \sigma_{12}, \sigma_{23}, \sigma_{31}\right)^{t}
$$

is a vector whose components are the atomic operators

$$
\sigma_{i j}=|i\rangle\langle j| .
$$

One obtains (9) from (13) through

$$
S_{\text {cond }}(\omega, \hat{\mathbf{k}} \lambda, t)=\sum_{i=1}^{3} \operatorname{tr}\left\{a_{\mathbf{k} \lambda}^{\dagger}(0) \sigma_{i i}(0) a_{\mathbf{k} \lambda}(0) \rho\left(t \mid t_{1}, t_{2}, \ldots\right)\right\} .
$$

Now we are going to derive the equations of motion of (13) for the $V$ system of Fig. 1, with one metastable upper level and driven by two lasers. The Hamiltonian in the Schrödinger picture for this system is given by [19]

$$
H=H_{\text {sys }}+H_{\Omega_{B}}+H_{\Omega_{S}},
$$

where

$$
\begin{aligned}
H_{\mathrm{sys}}= & \sum_{i=2}^{3} \hbar \omega_{i 1}|i\rangle\langle i|+\sum_{\mathbf{k} \lambda} \hbar \omega_{\mathbf{k} \lambda} a_{\mathbf{k} \lambda}^{\dagger} a_{\mathbf{k} \lambda} \\
& +\sum_{i=2}^{3} \frac{\hbar}{2} \Omega_{i}\left(|i\rangle\langle 1| e^{-i \tilde{\omega}_{i} t}+\text { H.c. }\right),
\end{aligned}
$$




$$
\begin{aligned}
& H_{\Omega_{B}}=\sum_{\mathbf{k} \lambda \in \Omega_{B}} \hbar\left(i g_{\mathbf{k} \lambda} a_{\mathbf{k} \lambda}|2\rangle\langle 1|+\text { H.c. }\right), \\
& H_{\Omega_{S}}=\sum_{\mathbf{k} \lambda \in \Omega_{S}} \hbar\left(i g_{\mathbf{k} \lambda} a_{\mathbf{k} \lambda}|2\rangle\langle 1|+\text { H.c. }\right) .
\end{aligned}
$$

Here $\Omega_{i}$ is the Rabi frequency of the laser $i$ with frequency $\tilde{\omega}_{i}$ driving the $i \leftrightarrow 1$ transition whose transition frequency is $\omega_{i 1}$. The coupling constant of the $2 \leftrightarrow 1$ transition to the quantized radiation field is given by

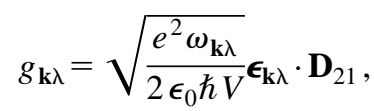

where $\boldsymbol{\epsilon}_{\mathbf{k} \lambda}$ is the polarization vector of the quantized mode $\mathbf{k} \lambda, \mathbf{D}_{i 1}=\langle i|\hat{\mathbf{x}}| 1\rangle$ is the transition dipole moment of the $i \leftrightarrow 1$ transition, and $V$ the quantization volume, later taken to infinity. It is convenient to go over to an interaction picture with respect to

$$
H_{0}=\sum_{i=2}^{3} \hbar \tilde{\omega}_{i}|i\rangle\langle i|+\sum_{\mathbf{k} \lambda} \hbar \omega_{\mathbf{k} \lambda} a_{\mathbf{k} \lambda}^{\dagger} a_{\mathbf{k} \lambda}
$$

We then obtain the Hamiltonian in the interaction picture as

$$
H_{I}=H_{\mathrm{sys}, I}+H_{\Omega_{B}, I}+H_{\Omega_{S}, I},
$$

with

$$
\begin{aligned}
& H_{\mathrm{sys}, I}=-\sum_{i=2}^{3} \hbar \Delta_{i}|i\rangle\langle i|+\sum_{i=2}^{3} \frac{\hbar}{2} \Omega_{i}(|i\rangle\langle 1|+| 1\rangle\langle i|), \\
& H_{\Omega_{B}, I}=\sum_{\mathbf{k} \lambda \in \Omega_{B}} \hbar\left(i g_{\mathbf{k} \lambda} a_{\mathbf{k} \lambda}|2\rangle\langle 1| e^{-i\left(\omega_{\mathbf{k} \lambda}-\tilde{\omega}_{2}\right) t}+\text { H.c. }\right), \\
& H_{\Omega_{S}, I}=\sum_{\mathbf{k} \lambda \in \Omega_{S}} \hbar\left(i g_{\mathbf{k} \lambda} a_{\mathbf{k} \lambda}|2\rangle\langle 1| e^{-i\left(\omega_{\mathbf{k} \lambda}-\tilde{\omega}_{2}\right) t}+\text { H.c. }\right)
\end{aligned}
$$

and the detuning

$$
\Delta_{i}=\tilde{\omega}_{i}-\omega_{i 1}
$$

\section{B. The state after the detection of a photon in the broadband detector}

First we simplify the state after the detection of a photon in the broadband detector which is given by (12). To this end we evaluate $U_{I}\left(t_{n}, s_{m_{n-1}}^{n-1}\right)$ in first order perturbation theory, insert it in (12), and keep only those contributions that are proportional to $\Delta t=t_{n}-s_{m_{n-1}}^{n-1}$. Since

$$
\left(1-\mathbb{P}_{0 \Omega_{B}}\right) H_{I}(t) \mathbb{P}_{0 \Omega_{B}}=\left(1-\mathbb{P}_{0 \Omega_{B}}\right) H_{I \mathrm{P}_{0 \Omega_{B}}}(t) \mathbb{P}_{0 \Omega_{B}}
$$

we find

$$
\begin{aligned}
\rho\left(t_{n}+0 \mid t_{1}, \ldots, t_{n-1}\right)= & \sum_{\mathbf{k} \lambda \in \Omega_{B}} g_{\mathbf{k} \lambda} 2 \int_{s_{m_{n-1}}^{n-1}}^{t_{n}} d t^{\prime} \int_{s_{m_{n-1}}^{n-1}}^{t_{n}} d t \\
& \times e^{\prime \prime\left(\omega_{\mathbf{k} \lambda}-\tilde{\omega}_{2}\right)\left(t^{\prime}-t^{\prime \prime}\right)} \sigma_{12}(0) \\
& \times \rho\left(s_{m_{n-1}-1}^{n-1} t_{1}, \ldots, t_{n-1}\right) \sigma_{21}(0) .
\end{aligned}
$$

In the Markov approximation this yields

$$
\rho\left(t_{n}+0 \mid t_{1}, \ldots, t_{n-1}\right)=C \rho\left(t_{n} \mid t_{1}, \ldots, t_{n-1}\right) C^{\dagger},
$$

with

$$
C:=\sqrt{2 \Gamma_{\Omega_{B}}} \sigma_{12}(0)
$$

and

$$
\Gamma_{\Omega_{B}}=\frac{e^{2} \omega_{21}^{3}}{16 \pi^{2} \hbar \epsilon_{0} c^{3}} \int_{\Omega_{B}} d \Omega\left(\left|\mathbf{D}_{21}\right|^{2}-\left|\hat{\mathbf{k}} \mathbf{D}_{21}\right|^{2}\right)
$$

If $\Omega_{B}$ is $4 \pi$, then $\Gamma_{\Omega_{B}}$ is just half the Einstein coefficient $A=2 \Gamma_{22}$ of the $2 \leftrightarrow 1$ transition. $\Gamma_{\Omega_{B}} / \Gamma_{22}$ is the average ratio of all scattered photons that are detected by the broadband detector. With (30) we have shown that the operations of taking the partial trace and multiplication with the projection operator $\mathbb{P}_{0 \Omega_{B}}$ can be abbreviated in Markov approximation by the action of $C$ and $C^{\dagger}$. This allows us to rewrite $\rho\left(t \mid t_{1}, t_{2}, \ldots\right)$, and upon inserting it into (13) we obtain for (13) the following form. To simplify notation we introduce for any operator $Q$ the operator $Q^{0}(t)$ by

$$
Q^{0}(t) \equiv U_{I}^{\dagger}(t, 0) \mathbb{P}_{0 \Omega_{B}} Q \mathrm{P}_{0 \Omega_{B}} U_{I}(t, 0)
$$

and use the subscript $M$ to label a certain fixed mode with wave vector $\mathbf{k}$ and polarization $\lambda$. With the abbreviation

$$
\mathscr{P}_{n}:=\prod_{j=0}^{m_{n}} \mathbb{P}_{0 \Omega_{B}}\left(s_{j}^{n}\right) \prod_{k=0}^{n-1}\left\{C\left(s_{0}^{k+1}\right) \prod_{i=0}^{m_{k}} \mathrm{P}_{0 \Omega_{B}}\left(s_{i}^{k}\right)\right\} \text {, }
$$

where $\mathrm{P}_{0 \Omega_{B}}(t)$ and $C(t)$ are defined similar to (33) without $\mathbb{P}_{0 \Omega_{B}}(t)$, we then find for (13)

$$
\begin{aligned}
\operatorname{tr}\left\{a_{M}^{\dagger}(0) \boldsymbol{\sigma}(0) a_{M}(0) \rho\left(t \mid t_{1}, t_{2}, \ldots\right)\right\} & \\
& =\left\langle\mathscr{P}_{n}^{\dagger}\left(a_{M}^{\dagger} \boldsymbol{\sigma} a_{M}\right)^{0}(t) \mathscr{P}_{n}\right\rangle .
\end{aligned}
$$

Note that the expectation value on the right is taken in the state $\rho(0)$ while the left hand side of (35) is the expectation value of $a_{M}^{\dagger}(0) \boldsymbol{\sigma}(0) a_{M}(0)$ in the state $\rho\left(t \mid t_{1}, t_{2}, \ldots\right)$.

\section{No-photon time evolution}

We are interested in the time evolution of (35) between successive detections and therefore we calculate the time derivative of the operator 


$$
\frac{d}{d t}\left(a_{M}^{\dagger} \boldsymbol{\sigma} a_{M}\right)^{0}(t)=\frac{i}{\hbar}\left[H_{I}(t),\left(a_{M}^{\dagger} \boldsymbol{\sigma} a_{M}\right)^{0}(t)\right],
$$

for which we will derive a Heisenberg-Langevin equation. Inserting (23) and using for $\mathbf{k} \in \Omega_{B}$ the relations

$$
a_{\mathbf{k} \lambda} \mathbb{P}_{0 \Omega_{B}}=\mathrm{P}_{0 \Omega_{B}} a_{\mathbf{k} \lambda}^{\dagger}=0
$$

one obtains

$$
\begin{aligned}
\frac{d}{d t}\left(a_{M}^{\dagger} \boldsymbol{\sigma} a_{M}\right)^{0}(t)= & \frac{i}{\hbar}\left[H_{\mathrm{sys}, I}(t),\left(a_{M}^{\dagger} \boldsymbol{\sigma} a_{M}\right)^{0}(t)\right]+\sum_{\mathbf{k} \lambda} g_{\mathbf{k} \lambda} e^{-i\left(\omega_{\mathbf{k} \lambda}-\tilde{\omega}_{2}\right) t}\left(a_{M}^{\dagger} \boldsymbol{\sigma} \sigma_{21} a_{M}\right)^{0}(t) a_{\mathbf{k} \lambda}(t) \\
& +\sum_{\mathbf{k} \lambda} g_{\mathbf{k} \lambda} e^{i\left(\omega_{\mathbf{k} \lambda}-\tilde{\omega}_{2}\right) t} a_{\mathbf{k} \lambda}^{\dagger}(t)\left(a_{M}^{\dagger} \sigma_{12} \boldsymbol{\sigma} a_{M}\right)^{0}(t)-\sum_{\mathbf{k} \lambda \in \Omega_{S}} g_{\mathbf{k} \lambda}\left(a_{M}^{\dagger} \sigma_{21} \boldsymbol{\sigma} a_{M}\right)^{0}(t) a_{\mathbf{k} \lambda}(t) e^{-i\left(\omega_{\mathbf{k} \lambda}-\tilde{\omega}_{2}\right) t} \\
& -\sum_{\mathbf{k} \lambda \in \Omega_{S}} g_{\mathbf{k} \lambda} a_{\mathbf{k} \lambda}^{\dagger}(t)\left(a_{M}^{\dagger} \boldsymbol{\sigma} \sigma_{12} a_{M}\right)^{0}(t) e^{i\left(\omega_{\mathbf{k} \lambda}-\tilde{\omega}_{2}\right) t}-g_{M}\left(\sigma_{21} \boldsymbol{\sigma} a_{M}\right)^{0}(t) e^{-i\left(\omega_{M}-\tilde{\omega}_{2}\right) t} \\
& -g_{M}\left(a_{M}^{\dagger} \boldsymbol{\sigma} \sigma_{12}\right)^{0}(t) a_{M}^{\dagger}(t) e^{i\left(\omega_{M}-\tilde{\omega}_{2}\right) t} .
\end{aligned}
$$

The last two terms already show that we will not find an equation for $\left(a_{M}^{\dagger} \boldsymbol{\sigma} a_{M}\right)^{0}(t)$ alone, but a coupled set of equations with the operators $\left(\boldsymbol{\sigma} a_{M}\right)^{0}(t), \quad\left(a_{M}^{\dagger} \boldsymbol{\sigma}\right)^{0}(t)$, and $(\boldsymbol{\sigma})^{0}(t)$. Since the calculations are all very similar we will not give them explicitly. Now one inserts

$$
a_{\mathbf{k} \lambda}(t)=a_{\mathbf{k} \lambda}(0)-g_{\mathbf{k} \lambda} \int_{0}^{t} d t^{\prime} \sigma_{12}\left(t^{\prime}\right) e^{i\left(\omega_{\mathbf{k} \lambda}-\tilde{\omega}_{2}\right) t^{\prime}}
$$

and its Hermitian conjugate into (38) and applies the Markov approximation. To simplify the notation we introduce the abbreviations

$$
\begin{gathered}
\mathbf{Q}_{1}(t)=(\boldsymbol{\sigma})^{0}(t), \\
\mathbf{Q}_{2}(t)=\left(\boldsymbol{\sigma} a_{M}\right)^{0}(t) e^{-i \Delta t}, \\
\mathbf{Q}_{3}(t)=\left(a_{M}^{\dagger} \boldsymbol{\sigma}\right)^{0}(t) e^{i \Delta t},
\end{gathered}
$$

$$
\mathbf{Q}_{4}(t)=\left(a_{M}^{\dagger} \boldsymbol{\sigma} a_{M}\right)^{0}(t),
$$

with $\Delta=\omega_{M}-\tilde{\omega}_{2}$, and the Langevin terms

$$
\begin{aligned}
\mathbf{F}_{i}(t)= & \mathbf{Q}_{i}(t) \sigma_{21}(t) E_{a}(t)+E_{c}(t) \sigma_{12}(t) \mathbf{Q}_{i}(t) \\
& -\sigma_{21}(t) \mathbf{Q}_{i}(t) E_{a \Omega_{S}}(t)-E_{c \Omega_{S}}(t) \mathbf{Q}_{i}(t) \sigma_{12}(t),
\end{aligned}
$$

with

$$
\begin{gathered}
E_{a}(t)=E_{c}^{\dagger}(t) \equiv \sum_{\mathbf{k} \lambda} g_{\mathbf{k} \lambda} a_{\mathbf{k} \lambda}(0) e^{-i\left(\omega_{\mathbf{k} \lambda}-\tilde{\omega}_{2}\right) t}, \\
E_{a \Omega_{S}}(t)=E_{c \Omega_{S}}^{\dagger}(t) \equiv \sum_{\mathbf{k} \lambda \in \Omega_{S}} g_{\mathbf{k} \lambda} a_{\mathbf{k} \lambda}(0) e^{-i\left(\omega_{\mathbf{k} \lambda}-\tilde{\omega}_{2}\right) t} .
\end{gathered}
$$

We define the $9 \times 9$ matrix $\mathbf{M}_{\Omega_{S}}$, with $\Delta_{32}=\Delta_{3}-\Delta_{2}$,

$$
\mathbf{M}_{\Omega_{S}}=\left(\begin{array}{ccccccccc}
0 & 2 \Gamma_{\Omega_{S}} & 0 & i \Omega_{2} / 2 & 0 & -i \Omega_{3} / 2 & -i \Omega_{2} / 2 & 0 & i \Omega_{3} / 2 \\
0 & -2 \Gamma_{22} & 0 & -i \Omega_{2} / 2 & 0 & 0 & i \Omega_{2} / 2 & 0 & 0 \\
0 & 0 & 0 & 0 & 0 & i \Omega_{3} / 2 & 0 & 0 & -i \Omega_{3} / 2 \\
i \Omega_{2} / 2 & -i \Omega_{2} / 2 & 0 & \Gamma_{22}-i \Delta_{2} & 0 & 0 & 0 & -i \Omega_{3} / 2 & 0 \\
0 & 0 & 0 & 0 & -\Gamma_{22}-i \Delta_{32} & 0 & -i \Omega_{3} / 2 & 0 & -i \Omega_{2} / 2 \\
-i \Omega_{3} / 2 & 0 & i \Omega_{3} / 2 & 0 & 0 & i \Delta_{3} & 0 & i \Omega_{2} / 2 & 0 \\
-i \Omega_{2} / 2 & i \Omega_{2} / 2 & 0 & 0 & -i \Omega_{3} / 2 & 0 & -\Gamma_{22}+i \Delta_{2} & 0 & 0 \\
0 & 0 & 0 & -i \Omega_{3} / 2 & 0 & i \Omega_{2} / 2 & 0 & -\Gamma_{22}+i \Delta_{32} & 0 \\
i \Omega_{3} / 2 & 0 & -i \Omega_{3} / 2 & 0 & -i \Omega_{2} / 2 & 0 & 0 & 0 & -i \Delta_{3}
\end{array}\right)
$$


and the matrices $\mathbf{V}$ and $\mathbf{W}$ whose coefficients are zero, except for

$$
\begin{array}{rll}
\mathbf{V}_{1,7}=-g_{M}, & \mathbf{V}_{4,1}=-g_{M}, & \mathbf{V}_{9,5}=-g_{M}, \\
\mathbf{W}_{1,4}=-g_{M}, & \mathbf{W}_{6,8}=-g_{M}, & \mathbf{W}_{7,2}=-g_{M} .
\end{array}
$$

We then find the Heisenberg-Langevin equations

$$
\begin{aligned}
\frac{d}{d t}\left(\begin{array}{l}
\mathbf{Q}_{1} \\
\mathbf{Q}_{2} \\
\mathbf{Q}_{3} \\
\mathbf{Q}_{4}
\end{array}\right)(t) & =\left(\begin{array}{cccc}
\mathbf{M}_{\Omega_{S}} & \mathbf{0} & \mathbf{0} & \mathbf{0} \\
\mathbf{V} & \mathbf{M}_{\Omega_{S}}-i \Delta 1 & \mathbf{0} & \mathbf{0} \\
\mathbf{W} & \mathbf{0} & \mathbf{M}_{\Omega_{S}}+i \Delta 1 & \mathbf{0} \\
\mathbf{0} & \mathbf{W} & \mathbf{V} & \mathbf{M}_{\Omega_{S}}
\end{array}\right) \\
& \times\left(\begin{array}{l}
\mathbf{Q}_{1} \\
\mathbf{Q}_{2} \\
\mathbf{Q}_{3} \\
\mathbf{Q}_{4}
\end{array}\right)(t)+\left(\begin{array}{c}
\mathbf{F}_{1} \\
\mathbf{F}_{2} \\
\mathbf{F}_{3} \\
\mathbf{F}_{4}
\end{array}\right)(t) \\
& \equiv \mathbf{L}_{\Omega_{S}} \mathbf{X}(t)+\mathbf{F}_{\Omega_{S}}(t),
\end{aligned}
$$

where we introduced the abbreviations $\mathbf{L}_{\Omega_{S}}, \mathbf{X}(t)$, and $\mathbf{F}_{\Omega_{S}}(t)$ to shorten notation. As already mentioned above there is no closed equation for $\left(a_{\mathbf{k} \lambda}^{\dagger} \boldsymbol{\sigma} a_{\mathbf{k} \lambda}\right)^{0}(t)$ alone, but a set of coupled equations together with $\left(a_{\mathbf{k} \lambda}^{\dagger} \boldsymbol{\sigma}\right)^{0}(t),\left(\boldsymbol{\sigma} a_{\mathbf{k} \lambda}\right)^{0}(t)$, and $(\boldsymbol{\sigma})^{0}(t)$.

We now insert the Heisenberg-Langevin equations into the time derivative of the right hand side of Eq. (35). We then find

$$
\frac{d}{d t}\left\langle\mathscr{P}_{n}^{\dagger} \mathbf{X}(t) \mathscr{P}_{n}\right\rangle=\mathbf{L}_{\Omega_{S}}\left\langle\mathscr{P}_{n}^{\dagger} \mathbf{X}(t) \mathscr{P}_{n}\right\rangle+\left\langle\mathscr{P}_{n}^{\dagger} \mathbf{F}_{\Omega_{S}}(t) \mathscr{P}_{n}\right\rangle .
$$

\section{Discussion of Langevin terms}

We now deal with the second contribution on the right hand side of (51). We are going to show that it is negligible, which will considerably simplify the integration of (51). Since $\mathbf{F}_{\Omega_{S}}(t)$ is linear in $a_{\mathbf{k} \lambda}(0)$ and $a_{\mathbf{k} \lambda}^{\dagger}(0)$ and the expectation value is taken in the state $\rho(0)$ given by (2) with the quantized radiation field in its vacuum state, $\left\langle\mathbf{F}_{\Omega_{S}}(t)\right\rangle$ vanishes. However, the vanishing of $\left\langle\mathscr{P}_{n}^{\dagger} \mathbf{F}_{\Omega_{S}}(t) \mathscr{P}_{n}\right\rangle$ is not obvious since the $a_{\mathbf{k} \lambda}(0)$ and $a_{\mathbf{k} \lambda}^{\dagger}(0)$ do not act on the vacuum state directly, but on the operator $\mathscr{P}_{n}$. In the following, however, we will show that it is possible in $\left\langle\mathscr{P}_{n}^{\dagger} \mathbf{F}_{\Omega_{S}}(t) \mathscr{P}_{n}\right\rangle$ to commute the $E_{a}(t)$ and $E_{a \Omega_{S}}(t)$ - which contain $a_{\mathbf{k} \lambda}(0)$ and $a_{\mathbf{k} \lambda}^{\dagger}(0)$ - to the right and the $E_{c}(t)$ and $E_{c \Omega_{S}}(t)$ to the left where they act on $\rho(0)$ and vanish. To this end we have to evaluate commutators with the operators $\mathrm{P}_{0 \Omega_{B}}\left(t^{\prime}\right)$ and $C\left(t^{\prime}\right)$ which occur in $\mathscr{P}_{n}$. We only show explicitly how to deal with the commutator

$$
\begin{aligned}
& {\left[\mathbf{E}_{a}(t), \mathbb{P}_{0 \Omega_{B}}\left(s_{2}\right)\right] \mathbb{P}_{0 \Omega_{B}}\left(s_{1}\right)} \\
& \quad=\sum_{\mathbf{k} \lambda} g_{\mathbf{k} \lambda} e^{-i\left(\omega_{\mathbf{k} \lambda}-\tilde{\omega}_{2}\right) t}\left[a_{\mathbf{k} \lambda}(0), \quad \mathbb{P}_{0 \Omega_{B}}\left(s_{2}\right)\right] \mathbb{P}_{0 \Omega_{B}}\left(s_{1}\right),
\end{aligned}
$$

where $s_{1}$ and $s_{2}$ are subsequent measurement times with $s_{2}-s_{1} \gg \omega_{21}^{-1}$.

Using (39) for $a_{\mathbf{k} \lambda}(0)$ we obtain

$$
\begin{aligned}
{\left[\mathbf{E}_{a}(t), \mathbb{P}_{0 \Omega_{B}}\left(s_{2}\right)\right] \mathbb{P}_{0 \Omega_{B}}\left(s_{1}\right)=} & \sum_{\mathbf{k} \lambda} g_{\mathbf{k} \lambda}{ }^{2} \int_{0}^{s_{2}} d t^{\prime} e^{-i\left(\omega_{\mathbf{k} \lambda}-\tilde{\omega}_{2}\right)\left(t-t^{\prime}\right)}\left[\sigma_{12}\left(t^{\prime}\right), \mathrm{P}_{0 \Omega_{B}}\left(s_{2}\right)\right] \\
& \times \mathbb{P}_{0 \Omega_{B}}\left(s_{1}\right)-\sum_{\mathbf{k} \lambda \in \Omega_{B}} g_{\mathbf{k} \lambda} \mathrm{P}_{0 \Omega_{B}}\left(s_{2}\right) a_{\mathbf{k} \lambda}\left(s_{2}\right) e^{-i\left(\omega_{\mathbf{k} \lambda}-\tilde{\omega}_{2}\right) t} \mathrm{P}_{0 \Omega_{B}}\left(s_{1}\right) .
\end{aligned}
$$

The integrand on the right hand side contains a function of the form

$$
\kappa\left(t-t^{\prime}\right) \equiv \sum_{\mathbf{k} \lambda} g_{\mathbf{k} \lambda} e^{-i\left(\omega_{\mathbf{k} \lambda}-\omega_{21}\right)\left(t-t^{\prime}\right)},
$$

which rapidly falls off in $t-t^{\prime}$ and is negligible for $\left|t-t^{\prime}\right| \gg \omega_{21}^{-1}$ [20]. Therefore we find that

$$
\Gamma_{22} g\left(t-s_{2}\right) \equiv \int_{0}^{s_{2}} d t^{\prime} \kappa\left(t-t^{\prime}\right)
$$

is negligible for $t-s_{2} \gg \omega_{21}^{-1}$, and $g(0)$ is of the order of 1 [20]. Since $\kappa\left(t-t^{\prime}\right)$ is peaked we also find

$$
\begin{aligned}
\int_{0}^{s_{2}} d t^{\prime} \kappa\left(t-t^{\prime}\right) & {\left[\sigma_{12}\left(t^{\prime}\right), \mathbb{P}_{0 \Omega_{B}}\left(s_{2}\right)\right] } \\
& \cong \Gamma_{22} g\left(t-s_{2}\right),\left[\sigma_{12}\left(s_{2}\right), \mathbb{P}_{0 \Omega_{B}}\left(s_{2}\right)\right]=0 .
\end{aligned}
$$

Inserting (39) into (53) again and using

$$
\int_{0}^{s_{1}} d t^{\prime} \kappa\left(t-t^{\prime}\right) \cong 0
$$

because of $t-s_{1} \gg \omega^{-1}$, we then find

$$
\begin{aligned}
{\left[E_{a}(t), \mathrm{P}_{0 \Omega_{B}}\left(s_{2}\right)\right] \mathrm{P}_{0 \Omega_{B}}\left(s_{1}\right) } & \\
& =\Gamma_{\Omega_{B}} g\left(t-s_{2}\right) \mathbb{P}_{0 \Omega_{B}}\left(s_{2}\right) \mathbb{P}_{0 \Omega_{B}}\left(s_{1}\right),
\end{aligned}
$$

and by analogous calculations

$$
\begin{aligned}
\mathbb{P}_{0 \Omega_{B}}\left(s_{1}\right)\left[\mathrm{P}_{0 \Omega_{B}}\left(s_{2}\right), E_{c}(t)\right] & \\
& =\Gamma_{\Omega_{B}} g\left(t-s_{2}\right) \mathrm{P}_{0 \Omega_{B}}\left(s_{2}\right) \mathrm{P}_{0 \Omega_{B}}\left(s_{1}\right),
\end{aligned}
$$




$$
\begin{aligned}
{\left[E_{a \Omega_{S}}(t), \mathrm{P}_{0 \Omega_{B}}\left(s_{2}\right)\right] } & \mathrm{P}_{0 \Omega_{B}}\left(s_{1}\right) \\
& =\Gamma_{\Omega_{S}} g\left(t-s_{2}\right) \mathbb{P}_{0 \Omega_{B}}\left(s_{2}\right) \mathbb{P}_{0 \Omega_{B}}\left(s_{1}\right),
\end{aligned}
$$

$$
\begin{aligned}
\mathrm{P}_{0 \Omega_{B}}\left(s_{1}\right)\left[\mathrm{P}_{0 \Omega_{B}}\left(s_{2}\right), E_{c \Omega_{S}}\right] & \\
& =\Gamma_{\Omega_{S}} g\left(t-s_{2}\right) \mathrm{P}_{0 \Omega_{B}}\left(s_{2}\right) \mathrm{P}_{0 \Omega_{B}}\left(s_{1}\right),
\end{aligned}
$$

$\left[E_{a}(t), C^{\dagger}\left(s_{2}\right)\right]=\Gamma_{22} g\left(t-s_{2}\right)\left(\sigma_{11}-\sigma_{22}\right)\left(s_{2}\right)$,

$\left[C\left(t_{2}\right), E_{c}(t)\right]=\Gamma_{22} g\left(t-s_{2}\right)\left(\sigma_{11}-\sigma_{22}\right)\left(s_{2}\right)$,

$\left[E_{a \Omega_{S}}(t), C^{\dagger}\left(t_{2}\right)\right]=\Gamma_{\Omega_{S}} g\left(t-s_{2}\right)\left(\sigma_{11}-\sigma_{22}\right)\left(s_{2}\right)$,

$\left[C\left(s_{2}\right), E_{c \Omega_{S}}(t)\right]=\Gamma_{\Omega_{S}} g\left(t-s_{2}\right)\left(\sigma_{11}-\sigma_{22}\right)\left(s_{2}\right)$.

Inserting these commutators in $\left\langle\mathscr{P}_{n}^{\dagger} \mathbf{F}_{\Omega_{S}}(t) \mathscr{P}_{n}\right\rangle$ we find that only a contribution proportional to $\Gamma_{22} g\left(t-s_{m_{n}}^{n}\right)$ survives. This can be neglected in the time evolution since $\Gamma_{22} g\left(t-s_{m_{n}}^{n}\right)$ is of the order of 1 for $t-s_{m_{n}}^{n}<\omega_{21}^{-1}$ and is negligible for $t-s_{m_{n}}^{n} \gg \omega_{21}^{-1}$ so that its contribution to $\left\langle\mathscr{P}_{n}^{\dagger} \mathbf{X}(t) \mathscr{P}_{n}\right\rangle$ for $t-s_{m_{n}}^{n} \gg \omega_{21}^{-1}$ is of the order of $\Gamma_{22} / \omega_{21}$, which is extremely small. Therefore we can neglect the Langevin term $\left\langle\mathscr{P}_{n}^{\dagger} \mathbf{X}(t) \mathscr{P}_{n}\right\rangle$ in the following.

Thus we have shown that the time evolution for (35), under the condition that no photon has been found in the broadband detector in the interval $\left(t_{n}, t\right]$, is given by

$$
\frac{d}{d t}\left\langle\mathscr{P}_{n}^{\dagger} \mathbf{X}(t) \mathscr{P}_{n}\right\rangle=\mathbf{L}_{\Omega_{S}}\left\langle\mathscr{P}_{n}^{\dagger} \mathbf{X}(t) \mathscr{P}_{n}\right\rangle .
$$

For the state $\left\langle\mathscr{P}_{n}^{\dagger} \mathbf{X}\left(t_{n}+0\right) \mathscr{P}_{n}\right\rangle$ after the detection of a photon at time $t_{n}$ in the broadband detector we find with (30), or directly from (35), $\left\langle\mathscr{P}_{n-1}^{\dagger} C^{\dagger}\left(t_{n}\right) \mathbf{X}\left(t_{n}\right) C\left(t_{n}\right) \mathscr{P}_{n-1}\right\rangle$. This can be rewritten as

$$
\left\langle\mathscr{P}_{n}^{\dagger} \mathbf{X}\left(t_{n}+0\right) \mathscr{P}_{n}\right\rangle=2 \Gamma_{\Omega_{B}} \mathbf{R}\left\langle\mathscr{P}_{n}^{\dagger} \mathbf{X}\left(t_{n}\right) \mathscr{P}_{n}\right\rangle,
$$

where $\mathbf{R}$ has matrix elements $\mathbf{R}_{1,2}=\mathbf{R}_{10,11}=\mathbf{R}_{19,20}$ $=\mathbf{R}_{28,29}=1$ and zero otherwise. If one only considers the time evolution of $\left\langle\mathscr{P}_{n}^{\dagger} \boldsymbol{\sigma}(t) \mathscr{P}_{n}\right\rangle$, i.e., for the state of the atom, and if one assumes a $4 \pi$-broadband counter, then (65) and (66) reduce to the ordinary quantum jump approach.

\section{E. The waiting-time distribution}

After the time evolution between detections in the broadband detector and the state after the detection we now determine the probability distribution for the detection times in the broadband detector, also called waiting-time distribution. We define the column vector $l$ by $l_{1}=l_{2}=l_{3}=1, l_{4}=\ldots$. $=l_{36}=0$, and the state

$$
\begin{aligned}
\mathbf{x}\left(t \mid t_{1}, t_{2}, \ldots\right) & \equiv\left\langle\mathscr{P}_{n}^{\dagger} \mathbf{X}(t) \mathscr{P}_{n}\right\rangle \\
& =e^{\mathbf{L}_{\Omega_{S}}{ }^{\left(t-t_{n}\right)}} \mathbf{R} e^{\mathbf{L}_{\Omega_{S}}{ }^{\left(t_{n}-t_{n-1}\right)}} \cdots \mathbf{R} e^{\mathbf{L}_{\Omega_{S}}{ }^{t_{1}} \mathbf{X}(0) .}
\end{aligned}
$$

The probability $P_{0}\left(t \mid t_{1}, \ldots, t_{n}\right)$ to find no photon in the interval $\left(t_{n}, t\right]$, under the condition that one has found photons at the times $t_{1}, \ldots, t_{n}$, is then given by

$$
\begin{aligned}
& P_{0}\left(t \mid t_{1}, \ldots, t_{n}\right) \\
& =\frac{\boldsymbol{l} \cdot e^{\mathbf{L}_{\Omega_{S}}\left(t-t_{n}\right)} \mathbf{R} e^{\mathbf{L}_{\Omega_{S}}\left(t_{n}-t_{n-1}\right)} \cdots \mathbf{R} e^{\mathbf{L}_{\Omega_{S} t_{1}} \mathbf{x}(0)}}{\boldsymbol{l} \cdot \mathbf{R} e^{\mathbf{L}_{\Omega_{S}}\left(t_{n}-t_{n-1}\right)} \cdots \mathbf{R} e^{\mathbf{L}_{\Omega_{S} t_{1}} \mathbf{x}(0)}} \\
& =\frac{\boldsymbol{l} \cdot \mathbf{x}\left(t \mid t_{1}, \ldots, t_{n}\right)}{\boldsymbol{l} \cdot \mathbf{R x}\left(t_{n} \mid t_{1}, \ldots, t_{n-1}\right)},
\end{aligned}
$$

which is just the trace over the atomic density matrix under the assumption that no photon has been found in $\left(t_{n}, t\right]$. The probability density for the detection of a photon at time $t$ is then given by

$$
\begin{aligned}
I_{1}\left(t \mid t_{1}, \ldots, t_{n}\right) & =-\frac{d}{d t} P_{0}\left(t \mid t_{1}, \ldots, t_{n}\right) \\
& =-\frac{\boldsymbol{l} \cdot \mathbf{L}_{\Omega_{S}} \mathbf{x}\left(t \mid t_{1}, \ldots, t_{n}\right)}{\boldsymbol{l} \cdot \mathbf{R x}\left(t_{n} \mid t_{1}, \ldots, t_{n-1}\right)} \\
& =2 \Gamma_{\Omega_{B}} \frac{\boldsymbol{l} \cdot \mathbf{R} \mathbf{R}\left(t \mid t_{1}, \ldots, t_{n}\right)}{\mathbf{R x}\left(t_{n} \mid t_{1}, \ldots, t_{n-1}\right)},
\end{aligned}
$$

where $\mathbf{L}_{\Omega_{S}}^{*} l=-2 \Gamma_{\Omega_{B}} \mathbf{R}^{*} l$ as well as $\Gamma_{22}-\Gamma_{\Omega_{S}}=\Gamma_{\Omega_{B}}$ have been used.

A simulation of a possible detection path runs as follows. The detection time $t_{n+1}$ is produced according to the probability density $I_{1}\left(t \mid t_{1}, \ldots, t_{n}\right)$, and the state $\left\langle\mathscr{P}_{n}^{\dagger} \mathbf{X}\left(t_{n+1}\right) \mathscr{P}_{n}\right\rangle$ at this time is calculated. With (66) one also obtains the state after the detection. This is repeated until a previously given time $t$ is reached. If one averages over infinitely many paths then one obtains

$$
\begin{aligned}
\mathbf{x}^{-}(t) & =P_{0}(t) \mathbf{x}(t)+\sum_{n=1}^{\infty} \int_{0}^{t} d t_{n} \ldots \int_{0}^{t_{2}} d t_{1} P_{0}\left(t \mid t_{1}, \ldots, t_{n}\right) I_{1}\left(t_{n} \mid t_{1}, \ldots, t_{n-1}\right) \cdots I_{1}\left(t_{1}\right) \mathbf{x}\left(t \mid t_{1}, \ldots, t_{n}\right) \\
& =e^{\mathbf{L}_{\Omega_{S}}{ }^{t} \mathbf{x}(0)+\sum_{n=1}^{\infty} \int_{0}^{t} d t_{n} \cdots \int_{0}^{t_{2}} d t_{1} e^{\mathbf{L}_{\Omega_{S}}\left(t-t_{n}\right)}\left(2 \Gamma_{\Omega_{B}} \mathbf{R}\right) \cdots\left(2 \Gamma_{\Omega_{B}} \mathbf{R}\right) e^{\mathbf{L}_{\Omega_{S}} t_{1}} \mathbf{x}(0)} \\
& =e^{\left(\mathbf{L}_{\Omega_{S}}+2 \Gamma_{\Omega_{B}} \mathbf{R}\right) t} \mathbf{x}(0)
\end{aligned}
$$


Since $\mathbf{L}_{\Omega_{S}}+2 \Gamma_{\Omega_{B}} \mathbf{R}$ is just the time evolution with no broadband detector present, we find that $\overline{\mathbf{x}}(t)$ is just the state of the complete ensemble at time $t$.

In the next section we will be interested in the calculation of the emission spectrum when the atom is in a light period of length at least $T$. This can be done as follows. We define a light period as a sequence of detection times where subsequent detections are separated by less than a given time $T_{0}$. A simulation of such a sequence would now proceed recursively as follows. If $t_{1}, \ldots, t_{n}$ have already been constructed, we produce a random detection time $t_{n+1}$ with the help of the probability density $I_{1}\left(t \mid t_{1}, \ldots, t_{n}\right)$ and calculate the state at time $t_{n+1}$ as above. If $t_{n+1} \geqslant T$ and $T-t_{n}<T_{0}$ we terminate the construction and save the sequence. If $t_{n+1} \geqslant T$ but $T-t_{n} \geqslant T_{0}$ we stop and discard the sequence. If $t_{n+1}<T$ we go on to produce $t_{n+2}$. Repeating this infinitely often and averaging over all saved results we obtain for $t \leqslant T$, instead of (70),

$$
\begin{aligned}
\overline{\mathbf{x}}_{L}(t)= & \left\{e^{\mathbf{L}_{\Omega_{S}}{ }^{t}} \chi_{T_{0}}(t)\right. \\
& +\sum_{n=1}^{\infty} \int_{0}^{t} d t_{n} \cdots \int_{0}^{t_{2}} d t_{1} e^{\mathbf{L}_{\Omega_{S}}\left(t-t_{n}\right)} \\
& \times\left(2 \Gamma_{\Omega_{B}} \mathbf{R}\right) \cdots\left(2 \Gamma_{\Omega_{B}} \mathbf{R}\right) e^{\mathbf{L}_{\Omega_{S}}{ }^{t_{1}}} \\
& \left.\times \chi_{T_{0}}\left(t-t_{n}\right) \cdots \chi_{T_{0}}\left(t_{1}\right)\right\} \mathbf{x}(0),
\end{aligned}
$$

where

$$
\chi_{T_{0}}(\tau) \equiv \begin{cases}1 & \text { for } \tau \leqslant T_{0} \\ 0 & \text { otherwise }\end{cases}
$$

This is the state, for $t \leqslant T$, of the subensemble which is in a light period (defined by $T_{0}$ ) of minimum length $T$.

With the simulation approaches explained above and the averaged results (70) and (71) to which they lead we are able to investigate the emission spectra of single radiating atoms.

From Eq. (71) it becomes apparent that the careful analysis given here is necessary because the result $\overline{\mathbf{x}}_{L}(t)$ depends on the decomposition of the operator $\mathbf{L}$ into $\mathbf{L}_{\Omega_{S}}$ and $\mathbf{R}$.

\section{F. Wave-function approach}

Until now we have calculated with the nine-component quantities, such as, for example, $\boldsymbol{\sigma}$ and their expectation values. That means that we deal with density matrices. In fact this is necessary since for a finite value of $\Omega_{S}$ the time evolution always produces mixed states since we trace over all possible events in the spectrometer. However, if one assumes a small spectrometer aperture, i.e., $\Omega_{S}$ small, then it is possible to simplify the calculations by working with wave functions, because then we may neglect $\Gamma_{\Omega_{S}}$. This can be seen as follows. For a given sequence of detection times, $t_{1}, t_{2}, \ldots$, in the broadband counter, we consider the threecomponent column vectors $\boldsymbol{\phi}(t)$ and $\boldsymbol{\psi}(t)$ satisfying, between detections, the equation

$$
\frac{d}{d t}\left(\begin{array}{c}
\boldsymbol{\phi} \\
\boldsymbol{\psi}
\end{array}\right)(t)=\left(\begin{array}{cc}
-i \mathbf{H}_{r e d} & \mathbf{0} \\
g_{M} \mathbf{C} & -i \mathbf{H}_{r e d}-i \Delta
\end{array}\right)\left(\begin{array}{c}
\boldsymbol{\phi} \\
\boldsymbol{\psi}
\end{array}\right)(t),
$$

where

$$
\mathbf{H}_{\text {red }}=\left(\begin{array}{ccc}
0 & \frac{\Omega_{2}}{2} & \frac{\Omega_{3}}{2} \\
\frac{\Omega_{2}}{2} & -i \Gamma_{22}-\Delta_{2} & 0 \\
\frac{\Omega_{3}}{2} & 0 & -\Delta_{3}
\end{array}\right), \quad \mathbf{C}=\left(\begin{array}{ccc}
0 & 1 & 0 \\
0 & 0 & 0 \\
0 & 0 & 0
\end{array}\right),
$$

and right after a detection

$$
\left(\begin{array}{l}
\boldsymbol{\phi} \\
\boldsymbol{\psi}
\end{array}\right)\left(t_{n}+0\right)=\sqrt{2 \Gamma_{22}}\left(\begin{array}{ll}
\mathbf{C} & \mathbf{0} \\
\mathbf{0} & \mathbf{C}
\end{array}\right)\left(\begin{array}{l}
\boldsymbol{\phi} \\
\boldsymbol{\psi}
\end{array}\right)\left(t_{n}\right) .
$$

In terms of $\phi_{i}^{*} \phi_{j}, \phi_{i}^{*} \psi_{j}, \psi_{i}^{*} \phi_{j}$, and $\psi_{i}^{*} \psi_{j}$ we then define the 36-column vector $\tilde{\mathbf{x}}\left(t \mid t_{1}, t_{2}, \ldots\right)$ by

$$
\begin{aligned}
\tilde{\mathbf{x}}\left(t \mid t_{1}, t_{2}, \ldots\right) & \\
& \equiv\left(\phi_{1}^{*} \phi_{1}, \ldots, \phi_{1}^{*} \psi_{1}, \ldots, \psi_{1}^{*} \phi_{1}, \ldots, \psi_{1}^{*} \psi_{1}, \ldots\right)^{t},
\end{aligned}
$$

where the ordering is that of Eq. (14). It now follows from Eqs. (73) and (75) by straightforward calculation for small $\Omega_{S}$ that $\tilde{\mathbf{x}}$ satisfies the same equations, Eqs. (65) and (66), as $\mathbf{x}\left(t \mid t_{1}, t_{2}, \ldots\right)$ does. Furthermore, if $\rho(0)$ is a pure state,

$$
\rho(0)=\left|0_{\gamma}\right\rangle\left|\varphi_{A}\right\rangle\left\langle\varphi_{A}\right|\left\langle 0_{\gamma}\right|,
$$

we choose as initial conditions

$$
\begin{gathered}
\phi_{i}(0)=\left\langle i \mid \varphi_{A}\right\rangle, \\
\psi_{i}(0)=0 .
\end{gathered}
$$

Then $\tilde{\mathbf{x}}$ satisfies the same initial condition as $\mathbf{x}$, as apparent from Eqs. (40)-(43), and since they also satisfy the same time evolution they coincide. Thus for small spectrometer aperture and pure initial state $\rho(0)$ it suffices to solve wavefunction equations.

Equations (73)-(75) have the same form as those in Ref. [12], but the derivation is completely different. Thus the expressions of Ref. [12] are special cases of (65) and (66) for a negligible spectrometer aperture. Unlike Ref. [12], we have derived (65) and (66) without having to invoke the theory of continuous measurement of Srinivas and Davies [21] to interpret the meaning of the equations. Also, the generalization of the theory to the description of different kinds of spectra is very straightforward in our approach. As an example we show how to extend it to absorption spectra.

\section{G. The absorption spectrum of a single atom}

Again we treat the $V$ system depicted in Fig. 1. The system is now probed by a weak laser which we model here by a mode $P$ which is in a coherent state of amplitude $\alpha_{P}$ at $t=0$. All other modes are initially assumed to be in the vacuum state. In order to apply the above theory we perform a unitary transformation which maps the initial state of the quantized radiation field onto the vacuum. This is achieved by the Mollow transformation 


$$
D(t) \equiv D\left(\alpha_{P} e^{-i \omega_{P} t}\right) \equiv \exp \left\{\alpha_{P} a_{p}^{\dagger} e^{-i \omega_{p} t}-\alpha_{P}^{*} a_{P} e^{i \omega_{p} t}\right\},
$$

for which

$$
\left|\alpha_{P} e^{-i \omega_{P} t}\right\rangle=D\left(\alpha_{P} e^{-i \omega_{P} t}\right)|0\rangle .
$$

We now turn to the transformed states

$$
\left|\psi_{D}(t)\right\rangle \equiv D^{\dagger}\left(\alpha_{P} e^{-i \omega_{P} t}\right)|\psi(t)\rangle,
$$

and in the new picture we go over to an interaction picture with respect to

$$
H_{0}=\sum_{i=2}^{3} \hbar \tilde{\omega}_{i}|i\rangle\langle i|+\sum_{\mathbf{k} \lambda} \hbar \omega_{\mathbf{k} \lambda} a_{\mathbf{k} \lambda}^{\dagger} a_{\mathbf{k} \lambda} .
$$

We then find the new Hamiltonian

$$
H_{I}=-\sum_{i=2}^{3} \hbar \Delta_{i}|i\rangle\langle i|+\sum_{i=2}^{3} \frac{\hbar}{2} \Omega_{i}(|i\rangle\langle 1|+| 1\rangle\langle i|)
$$

$$
\begin{aligned}
& +\sum_{\mathbf{k} \lambda} \hbar\left\{i g_{\mathbf{k} \lambda} a_{\mathbf{k} \lambda}|2\rangle\left\langle 1\left|e^{-i\left(\omega_{\mathbf{k} \lambda}-\tilde{\omega}_{2}\right) t}-i g_{\mathbf{k} \lambda} a_{\mathbf{k} \lambda}^{\dagger}\right| 1\right\rangle\right. \\
& \left.\quad \times\langle 2| e^{+i\left(\omega_{\mathbf{k} \lambda}-\tilde{\omega}_{2}\right) t}\right\} \\
& +\hbar \frac{\Omega_{P}}{2}|2\rangle\left\langle 1\left|e^{-i\left(\omega_{P}-\tilde{\omega}_{2}\right) t}+\hbar \frac{\Omega_{P}^{*}}{2}\right| 1\right\rangle\langle 2| e^{i\left(\omega_{P}-\tilde{\omega}_{2}\right) t},
\end{aligned}
$$

where $\Omega_{P}=2 i g_{P} \alpha_{P}$. Now the approach derived in this section applies since in the transformed picture the initial state is $\rho_{D}(0)=\rho_{A} \otimes\left|0_{\gamma}\right\rangle\left\langle 0_{\gamma}\right|$. Because the only change in the Hamiltonian is an additional classical field in (81) compared to (23), we immediately obtain for the time evolution between successive detections

$$
\frac{d}{d t}\left(\begin{array}{c}
\left\langle\mathscr{P}_{n}^{\dagger} \mathbf{Q}_{1}(t) \mathscr{P}_{n}\right\rangle \\
\left\langle\mathscr{P}_{n}^{\dagger} \mathbf{Q}_{2}(t) \mathscr{P}_{n}\right\rangle \\
\left\langle\mathscr{P}_{n}^{\dagger} \mathbf{Q}_{3}(t) \mathscr{P}_{n}\right\rangle \\
\left\langle\mathscr{P}_{n}^{\dagger} \mathbf{Q}_{4}(t) \mathscr{P}_{n}\right\rangle
\end{array}\right)=\left(\begin{array}{cccc}
\mathbf{M}_{\Omega_{S}}(t) & \mathbf{0} & \mathbf{0} & \mathbf{0} \\
\mathbf{V} & \mathbf{M}_{\Omega_{S}}(t)-i \Delta 1 & \mathbf{0} & \mathbf{0} \\
\mathbf{W} & \mathbf{0} & \mathbf{M}_{\Omega_{S}}(t)+i \Delta 1 & \mathbf{0} \\
\mathbf{0} & \mathbf{W} & \mathbf{V} & \mathbf{M}_{\Omega_{S}}(t)
\end{array}\right)\left(\begin{array}{c}
\left\langle\mathscr{P}_{n}^{\dagger} \mathbf{Q}_{1}(t) \mathscr{P}_{n}\right\rangle \\
\left\langle\mathscr{P}_{n}^{\dagger} \mathbf{Q}_{2}(t) \mathscr{P}_{n}\right\rangle \\
\left\langle\mathscr{P}_{n}^{\dagger} \mathbf{Q}_{3}(t) \mathscr{P}_{n}\right\rangle \\
\left\langle\mathscr{P}_{n}^{\dagger} \mathbf{Q}_{4}(t) \mathscr{P}_{n}\right\rangle
\end{array}\right),
$$

where

$$
\mathbf{M}_{\Omega_{S}}(t)=\mathbf{M}_{\Omega_{S}}+\mathbf{P}(t),
$$

with

$$
\mathbf{P}(t)=\left(\begin{array}{ccccccccc}
0 & 0 & 0 & f(t) & 0 & 0 & f^{*}(t) & 0 & 0 \\
0 & 0 & 0 & -f(t) & 0 & 0 & -f^{*}(t) & 0 & 0 \\
0 & 0 & 0 & 0 & 0 & 0 & 0 & 0 & 0 \\
-f^{*}(t) & f^{*}(t) & 0 & 0 & 0 & 0 & 0 & 0 & 0 \\
0 & 0 & 0 & 0 & 0 & 0 & 0 & 0 & -f(t) \\
0 & 0 & 0 & 0 & 0 & 0 & 0 & f(t) & 0 \\
-f(t) & f(t) & 0 & 0 & 0 & 0 & 0 & 0 & 0 \\
0 & 0 & 0 & 0 & 0 & -f^{*}(t) & 0 & 0 & 0 \\
0 & 0 & 0 & 0 & f^{*}(t) & 0 & 0 & 0 & 0
\end{array}\right)
$$

and

$$
f(t)=i \frac{\Omega_{P}}{2} e^{i \Delta t} .
$$

The contribution $\mathbf{P}(t)$ is due to the additional classical field in the Hamiltonian (81). We immediately obtain for the state after the detection of a photon

$$
\left(\begin{array}{c}
\left\langle\mathscr{P}_{n}^{\dagger} \mathbf{Q}_{1}\left(t_{n}+0\right) \mathscr{P}_{n}\right\rangle \\
\left\langle\mathscr{P}_{n}^{\dagger} \mathbf{Q}_{2}\left(t_{n}+0\right) \mathscr{P}_{n}\right\rangle \\
\left\langle\mathscr{P}_{n}^{\dagger} \mathbf{Q}_{3}\left(t_{n}+0\right) \mathscr{P}_{n}\right\rangle \\
\left\langle\mathscr{P}_{n}^{\dagger} \mathbf{Q}_{4}\left(t_{n}+0\right) \mathscr{P}_{n}\right\rangle
\end{array}\right)=2 \Gamma_{\Omega_{B}}\left(\begin{array}{cccc}
\mathbf{R} & \mathbf{0} & \mathbf{0} & \mathbf{0} \\
\mathbf{0} & \mathbf{R} & \mathbf{0} & \mathbf{0} \\
\mathbf{0} & \mathbf{0} & \mathbf{R} & \mathbf{0} \\
\mathbf{0} & \mathbf{0} & \mathbf{0} & \mathbf{R}
\end{array}\right)\left(\begin{array}{c}
\left\langle\mathscr{P}_{n}^{\dagger} \mathbf{Q}_{1}\left(t_{n}\right) \mathscr{P}_{n}\right\rangle \\
\left\langle\mathscr{P}_{n}^{\dagger} \mathbf{Q}_{2}\left(t_{n}\right) \mathscr{P}_{n}\right\rangle \\
\left\langle\mathscr{P}_{n}^{\dagger} \mathbf{Q}_{3}\left(t_{n}\right) \mathscr{P}_{n}\right\rangle \\
\left\langle\mathscr{P}_{n}^{\dagger} \mathbf{Q}_{4}\left(t_{n}\right) \mathscr{P}_{n}\right\rangle
\end{array}\right)
$$


since in its derivation the classical fields are irrelevant. As for the emission spectrum it is possible to go over to a wavefunction description as in (73)-(75) if the solid angle covered by the spectrometer is negligible, i.e., in the case of the absorption spectrum the divergence of the laser has to be small because we are now interested only in the weak laser.

We define the absorption spectrum by the change $\Delta N(t)$ of the expectation value of the photon number in the probe, divided by the elapsed time $t$,

$$
\Delta N(t)=\frac{N(t)-N(0)}{t} .
$$

If $\Delta N(t)>0$ one has amplification, if $\Delta N(t)<0$ one has absorption of the probe. For the calculation of $\Delta N(t)$ we need the photon number operator $\hat{N}$ in the transformed picture. One obtains

$$
\begin{aligned}
\hat{N}(t) & =U_{0}^{\dagger}(t, 0) D^{\dagger}\left(\alpha_{P} e^{-i \omega_{P} t}\right) a_{P}^{\dagger} a_{P} D\left(\alpha_{P} e^{-i \omega_{P} t}\right) U_{0}(t, 0) \\
& =a_{P}^{\dagger} a_{P}+a_{P}^{\dagger} \alpha_{P}+a_{P} \alpha_{P}^{*}+\left|\alpha_{P}\right|^{2}
\end{aligned}
$$

From this we find the photon number in the probe mode to be

$$
\begin{aligned}
N(t)= & \sum_{i=1}^{3}\left\{\left|\alpha_{P}\right|^{2} X_{i}(t)+X_{27+i}(t)+\alpha_{P} X_{18+i}(t)\right. \\
& \left.+\alpha_{P}^{*} X_{9+i}(t)\right\} .
\end{aligned}
$$

It can be shown that for sufficiently large times the absorption spectrum defined by (86) coincides with

$$
A(\Delta)=\operatorname{Re} \frac{\Omega_{P}^{2}}{2} \int_{0}^{\infty} d \tau e^{i \Delta \tau}\left\langle\left[\sigma_{21}(\tau), \sigma_{12}(0)\right]\right\rangle_{s s},
$$

which can be found, for example, in Ref. [22].

Therefore we have shown how to derive simulation equations for the absorption spectrum from the basic definition (86), instead of starting from the final result (89) as, for example, in Ref. [12]. The treatment of the absorption spectrum also shows how different nonclassical probe beams can be incorporated. For example, starting with an initially squeezed probe mode we may go over via the squeezing operator to a picture where the mode is in the vacuum state and apply the formalism again.

As a last extension of the treatment of the resonancefluorescence spectrum we turn to the higher order correlations between different photon number states of the mode $\mathbf{M}$. They are described by the quantities

$$
\mathbf{E}_{l, m}(t) \equiv \operatorname{tr}\left\{\left(a_{M}^{\dagger}\right)^{l} \boldsymbol{\sigma}\left(a_{M}\right)^{m} e^{i(l-m) \Delta t} \rho\left(t \mid t_{1}, t_{2}, \ldots\right)\right\} .
$$

The time evolution between successive detections in the broadband detector is given by

$$
\begin{aligned}
\frac{d}{d t} \mathbf{E}_{l, m}(t)= & \left\{\mathbf{M}_{\Omega_{S}}+i(l-m) \Delta\right\} \mathbf{E}_{l, m}(t)+m g_{M} \mathbf{V} \mathbf{E}_{l, m-1}(t) \\
& +l g_{M} \mathbf{W} \mathbf{E}_{l-1, m}(t)
\end{aligned}
$$

and the state right after detection at $t_{n}$ is given by

$$
\mathbf{E}_{l, m}\left(t_{n}+0\right)=2 \Gamma_{\Omega_{B}} \mathscr{B} \mathbf{E}_{l, m}\left(t_{n}\right) .
$$

After having shown how to derive the correct simulation equations for the resonance-fluorescence spectrum of a single atom and possible extensions to other spectra we are now going to apply the theory to the spectrum of resonance fluorescence of a $V$ system with one metastable transition. The results are presented in the next section.

\section{THE RESONANCE-FLUORESCENCE SPECTRUM OF A SINGLE $V$ SYSTEM}

In Sec. II of this paper we have derived simulation equations for atoms on which both time-resolved as well as spectrally resolved measurements are performed simultaneously. We now turn to an application of the theory. We investigate the spectrum of resonance fluorescence of a $V$ system with one metastable transition and irradiated by two lasers (see Fig. 1). For suitable parameters such a system is known to exhibit light and dark periods in its resonance fluorescence. We are interested both in the spectrum of resonance fluorescence of the complete ensemble as well as that for a single $V$ system in a light period. In the following we assume that the spectrometer is very small $\left(\Omega_{S}\right.$ is negligible) so that we can use Eqs. (73) - (75) instead of the more general Eqs. (65)(66). This has the advantage that the simulations are much faster because Eqs. (73)-(75) describe the time evolution of a state vector with six elements while Eqs. (65) and (66) deal with a vector with 36 components.

The spectrum of resonance fluorescence of an ensemble of $V$ systems with one metastable transition driven by two lasers has recently been discussed by us [9]. We give here the analytic expressions and a brief discussion. For details we refer to Ref. [9]. We assume that the conditions

$$
\Omega_{3}^{2} \ll \frac{1}{4} \frac{16 \Delta_{3}^{2} \Gamma_{22}^{2}+\left[\Omega_{2}^{2}+4 \Delta_{3}\left(\Delta_{2}-\Delta_{3}\right)\right]^{2}}{\Gamma_{22}^{2}+\left(\Delta_{2}-\Delta_{3}\right)^{2}}
$$

and

$$
\Gamma_{33} \ll \frac{\Omega_{2}^{2} \Omega_{3}^{2} \Gamma_{22}}{16 \Delta_{3}^{2} \Gamma_{22}^{2}+\left\{\Omega_{2}^{2}+4 \Delta_{3}\left(\Delta_{2}-\Delta_{3}\right)\right\}^{2}}
$$

are satisfied. In this parameter range intermittent fluorescence occurs and stimulated transitions on the $3 \leftrightarrow 1$ dominate Eq. (94). The normalized spectrum of resonance fluorescence $S(\Delta)$ of the strong $2 \leftrightarrow 1$ transition is given by

$$
\begin{aligned}
S(\Delta) & =\frac{1}{\pi \rho_{22}^{\mathrm{SS}}} \operatorname{Re} \int_{0}^{\infty} d \tau\left\langle\sigma_{21}(\tau) \sigma(0)\right\rangle_{\mathrm{SS}} e^{-i \Delta \tau} \\
& =S_{\text {Mollow }}(\Delta)+S_{\text {coh }}(\Delta)+S_{\text {peak }}(\Delta),
\end{aligned}
$$

where the expectation value is performed in the steady state of the atom and $\rho_{22}^{\mathrm{SS}}$ is the steady-state population in level 2. With the abbreviations

$$
\begin{gathered}
B=-2 \Omega_{2}^{2}+6 \Gamma_{22}^{2}-2 \Delta_{2}^{2}, \\
C=\Omega_{2}^{4}+2 \Omega_{2}^{2} \Gamma_{22}^{2}+9 \Gamma_{22}^{4}+\Delta_{2}^{4}+2 \Delta_{2}^{2} \Omega_{2}^{2}-6 \Gamma_{22}^{2} \Delta_{2}^{2},
\end{gathered}
$$

$$
D=\Gamma_{22}^{2}\left(\Omega_{2}^{2}+2 \Delta_{2}^{2}+2 \Gamma_{22}^{2}\right)^{2}
$$




$$
\begin{gathered}
A_{p}=2 \frac{\left(\Delta_{2}^{2}+\Gamma_{22}^{2}\right)\left[\left(\Delta_{2}-\Delta_{3}\right)^{2}+\Gamma_{22}^{2}\right]\left[\left(\Omega_{2}^{2}-4 \Delta_{3}^{2}+4 \Delta_{2} \Delta_{3}\right)^{2}+16 \Delta_{3}^{2} \Gamma_{22}^{2}\right]}{\Omega_{2}^{2} \Omega_{3}^{2} \Gamma_{22}\left[\Omega_{2}^{2}+2 \Delta_{3}^{2}+4 \Delta_{2}^{2}-4 \Delta_{2} \Delta_{3}+4 \Gamma_{22}^{2}\right]^{2}}, \\
\Gamma_{p}=\frac{2 \Omega_{2}^{2} \Omega_{3}^{2} \Gamma_{22}\left(2 \Delta_{3}^{2}+4 \Delta_{2}^{2}-4 \Delta_{2} \Delta_{3}+\Omega_{2}^{2}+4 \Gamma_{22}^{2}\right)}{\left[\left(\Omega_{2}^{2}-4 \Delta_{3}^{2}+4 \Delta_{2} \Delta_{3}\right)^{2}+16 \Delta_{3}^{2} \Gamma_{22}^{2}\right]\left(\Omega_{2}^{2}+2 \Delta_{2}^{2}+2 \Gamma_{22}^{2}\right)},
\end{gathered}
$$

one then obtains, under the conditions (93) and (94), the approximate result

$$
\begin{gathered}
\pi S_{\text {Mollow }}(\Delta)=\frac{\Gamma_{22} \Omega_{2}^{2}\left(\Omega_{2}^{2}+2 \Delta^{2}+8 \Gamma_{22}^{2}\right)}{2\left(\Delta^{6}+B \Delta^{4}+C \Delta^{2}+D\right)} \\
\pi S_{\text {coh }}(\Delta)=\pi \frac{2\left(\Delta_{2}^{2}+\Gamma_{22}^{2}\right)}{4 \Gamma_{22}^{2}+\Omega_{2}^{2}+2 \Delta_{3}^{2}+4 \Delta_{2}^{2}-4 \Delta_{2} \Delta_{3}} \delta(\Delta) \\
\pi S_{\text {peak }}(\Delta)=\frac{A_{p} \Gamma_{p}^{2}}{\Delta^{2}+\Gamma_{p}^{2}}
\end{gathered}
$$

for the normalized spectrum of resonance fluorescence. There are three distinct contributions. $S_{\text {Mollow }}(\Delta)$ is the wellknown three-peaked Mollow spectrum, $S_{\text {coh }}(\Delta)$ is the Rayleigh peak and $S_{\text {peak }}(\Delta)$ is an extremely narrow peak in the resonance-fluorescence spectrum of a $V$ system which we have recently discussed in Ref. [9]. The width $\Gamma_{p}$ of this narrow peak is just the sum of the inverse lengths of light and dark periods, $\Gamma_{p}=1 / T_{D}+1 / T_{L}$, that occur in the intensity of resonance fluorescence of the strong $2 \leftrightarrow 1$ transition. In Fig. 3 we have plotted the spectrum of resonance fluorescence for the parameters $\Omega_{2}=2 \Gamma_{22}, \Omega_{3}=0.2 \Gamma_{22}$, and $\Delta_{2}=\Delta_{3}=0$. The laser driving the $2 \leftrightarrow 1$ transition is not sufficiently strong for sidebands in the spectrum to become visible [23], whereas the narrow peak is easily seen in this plot. The origin of the peak can be traced back to the modulation of the resonance-fluorescence due to light and dark periods. A simplified model of resonance fluorescence simulating this modulation by a classical stochastic process is able to reproduce the narrow peak in the resonance-fluorescence spectrum. For a more detailed account of this see Ref. [9].

In the following we are going to investigate the resonance-fluorescence spectrum of the $V$ system for the parameters given in Fig. 3, but now instead of calculating the spectrum of resonance fluorescence for the complete ensemble we restrict ourselves to $V$ systems in a light period. For this purpose we need the theory developed in Sec. II. At first glance one is tempted to expect that the spectrum of resonance fluorescence will not change when observed only in a long light period because, intuitively, all photons are emitted in a light period. This, however, turns out to be not true. In the following we will exhibit this numerically and give a physical explanation for this behavior.

In our simulation we slightly generalize the notion of a light period in a way suitable for our purposes. Given a time difference $T_{0}$ we call a sequence of photon emissions a light period if the time interval between subsequent emissions is always smaller than $T_{0}$. A time interval between successive emissions that is longer than $T_{0}$ is then called a dark period.
Usually the time constant $T_{0}$ is chosen to be a few lifetimes of the fluorescing level [here level 2 with lifetime $\left.1 /\left(2 \Gamma_{22}\right)\right]$. However, we would like to choose arbitrary values for $T_{0}$ because this enables us to investigate the transition from the complete ensemble $\left(T_{0}=\infty\right)$ to the ensemble in a light period ( $T_{0}$ is equal to a few lifetimes of level 2$)$ by simply changing $T_{0}$.

With the help of $P_{0}(t)$ and $I_{1}(t)$ given in (68) and (69) and for a given maximal waiting time $T_{0}$, we find for the mean length of a dark period

$$
T_{D}\left(T_{0}\right)=\frac{\int_{T_{0}}^{\infty} d t^{\prime} t^{\prime} I_{1}\left(t^{\prime}\right)}{\int_{T_{0}}^{\infty} d t^{\prime} I_{1}\left(t^{\prime}\right)}=T_{0}+\frac{\int_{T_{0}}^{\infty} d t^{\prime} P_{0}\left(t^{\prime}\right)}{P_{0}\left(T_{0}\right)}
$$

For $T_{0} \gg \Gamma_{22}^{-1}$ the second term is easily evaluated. Then in the integrand only a single exponential of the form $p \exp \left\{-2\left|\operatorname{Im} \lambda_{1}\right| t^{\prime}\right\}$ is relevant where $\lambda_{1}$ is the eigenvalue of $\mathbf{H}_{\text {red }}$ in (74) with smallest imaginary part. All other exponentials in $P_{0}\left(t^{\prime}\right)$ have essentially dropped off to 0 so that $P_{0}\left(t^{\prime}\right)$ is of the form

$$
P_{0}\left(t^{\prime}\right)=p e^{-\left|2 \operatorname{Im} \lambda_{1}\right| t} \text { for } t>T_{0} \gg \Gamma_{22}^{-1} .
$$

Thus one obtains

$$
T_{D}\left(T_{0}\right)=T_{0}+\frac{1}{\left|2 \operatorname{Im} \lambda_{1}\right|} \equiv T_{0}+T_{D}
$$

An analytical expression for $T_{D}$, valid under the conditions (93) and (94), is the eigenvalue with the smallest imaginary part of $\mathbf{H}_{\text {red }}$ and is given in (109) below. The mean time between successive emissions in a light period is

$$
\tau_{L}\left(T_{0}\right)=\frac{\int_{0}^{T_{0}} d t^{\prime} t^{\prime} I_{1}\left(t^{\prime}\right)}{\int_{0}^{T_{0}} d t^{\prime} I_{1}\left(t^{\prime}\right)}
$$

and thus the mean length of a light period, for given $T_{0}$, is

$$
\begin{aligned}
T_{L}\left(T_{0}\right) & =\frac{\tau_{L}\left(T_{0}\right)}{P_{0}\left(T_{0}\right)}=\frac{\tau_{L}\left(T_{0}\right)}{p} e^{T_{0} / T_{D}} \\
& =\left(T_{D}+T_{L}\right) e^{T_{0} / T_{D}}-\left(T_{D}+T_{0}\right)
\end{aligned}
$$

for $P_{0}\left(T_{0}\right) \ll 1$. If both light and dark periods have mean lengths which are much longer than the lifetime of level 2, then one can make the usual choice $T_{0} \ll T_{D}, T_{L}$ and one has $T_{D}\left(T_{0}\right)=T_{D}$ and $T_{L}\left(T_{0}\right)=T_{L}$. For the $V$ system we find under the conditions of Eqs. (93) and (94) 


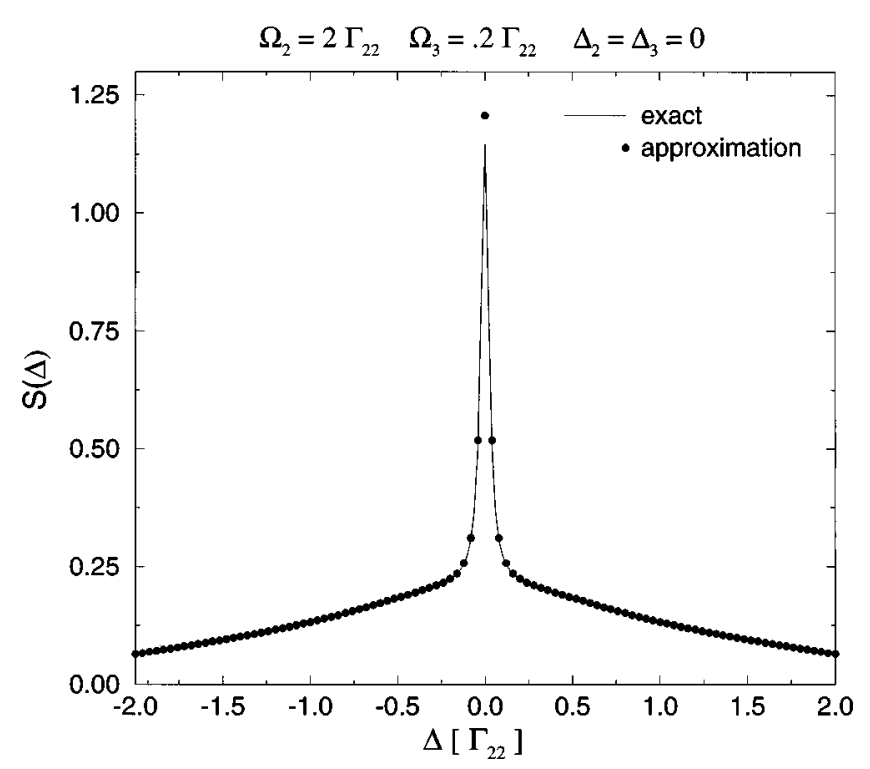

FIG. 3. The steady-state spectrum of resonance fluorescence for an ensemble of $V$ systems for $\Omega_{2}=2 \Gamma_{22}, \Omega_{3}=0.2 \Gamma_{22}$, and $\Delta_{2}=\Delta_{3}=0$. There is a narrow peak at the origin. The approximate results (dots) coincide very well with the exact spectrum (solid line).

$$
\begin{gathered}
T_{D}=\frac{16 \Delta_{3}^{2} \Gamma_{22}^{2}+\left[\Omega_{2}^{2}-4 \Delta_{3}\left(\Delta_{3}-\Delta_{2}\right)\right]^{2}}{2 \Omega_{2}^{2} \Omega_{3}^{2} \Gamma_{22}}, \\
T_{L}=\frac{2 \Delta_{2}^{2}+2 \Gamma_{22}^{2}+\Omega_{2}^{2}}{2 \Gamma_{22}^{2}+2\left(\Delta_{2}-\Delta_{3}\right)^{2}} T_{D} .
\end{gathered}
$$

For the parameters used in Fig. 3 we then obtain

$$
\begin{aligned}
& T_{D}=50 \Gamma_{22}^{-1}, \\
& T_{L}=150 \Gamma_{22}^{-1} .
\end{aligned}
$$

We now turn to the simulations of the resonancefluorescence spectrum. First we choose $T_{0}=\infty$ so that we should obtain the resonance-fluorescence spectrum of the complete ensemble. In fact, a simulation for the parameters $\Omega_{2}=2 \Gamma_{22}, \Omega_{3}=0.2 \Gamma_{22}$, and $\Delta_{2}=\Delta_{3}=0$ with 1000 runs, shown in Fig. 4, exhibits very good agreement of the simulation results (dots) with the exact results (solid line). The small deviations are due to the finite number of runs which results in an uncertainty of about $3 \%$.

After having shown that the simulation is able to reproduce the resonance-fluorescence spectrum of the complete ensemble we now investigate the transition from the complete ensemble $\left(T_{0}=\infty\right)$ to the ensemble in a light period $\left(T_{0}=50 \Gamma_{22}\right)$. In Fig. 5 we have plotted the spectrum of resonance fluorescence for the same set of parameters as in Figs. 3 and 4 , but now for finite values of $T_{0}$, i.e., $T_{0}=50 \Gamma_{22}^{-1}, 75 \Gamma_{22}^{-1}, 100 \Gamma_{22}^{-1}, 150 \Gamma_{22}^{-1}, 200 \Gamma_{22}^{-1}$. The simulation time for each run was $T=15000 \Gamma_{22}^{-1}$, i.e., we assumed a light period of at least this length. Of course, light periods of such a length, for example, with $T_{0}=50 \Gamma_{22}^{-1}$, are rare, but we wanted to first treat the case where spectral broadening of the Rayleigh peak due to the finite measurement time is neg- ligible. In Fig. 5 one sees that the amplitude of the narrow peak rapidly decreases with diminishing values of $T_{0}$. Therefore, by observing the resonance-fluorescence spectrum exclusively in a light period, one destroys the narrow peak in the spectrum. One also sees from Fig. 5 that only the narrow central peak is significantly affected by the restriction to light periods. For $\Delta \geqslant 0.1 \Gamma_{22}$ the spectra for different $T_{0}$ coincide almost exactly. Because of the long measurement time $T$ chosen here the vanishing of the narrow central peak is not due to a broadening of the spectrum by the time-energy uncertainty relation.

Since light periods with lengths $T=15000 \Gamma_{22}^{-1}$ are rare we also present results for light periods whose lengths are at least $T=2800 \Gamma_{22}^{-1}, T=1500 \Gamma_{22}^{-1}$, or $T=1000 \Gamma_{22}^{-1}$. From the fact that the lengths of the light and dark periods approximately obey a Poissonian distribution we easily find that light periods of length of at least $T=1000 \Gamma_{22}^{-1}$ are quite probable. For example, for $T_{0}=75 \Gamma_{22}^{-1}$ the probability for a light period with length exceeding $T=1000 \Gamma_{22}^{-1}$ is 0.268 , which is quite large. Of course, in these cases we have, due to the shorter measurement intervals (length is at least $T$ ), a noticeable broadened Rayleigh peak whose width is essentially proportional to $1 / T$. But as one sees from Figs. 6-8, the narrow peak is still there and also its characteristic dependence on $T_{0}$ is clearly visible.

These results can now immediately be carried over to a single $V$ system. For suitable parameters such a system exhibits, when monitored by a broadband detector in the solid angle $\Omega_{B}$, intermittent light and dark periods. Triggered by the detector, one can analyze the spectrum in another solid angle $\Omega_{S}$ for sufficiently long light periods at a time $T$ after their respective beginning. The mean of these spectra coincides with what we have calculated.

In this section we have presented simulation results for the resonance-fluorescence spectrum in a light period and have shown that the original narrow peak in the spectrum of the complete ensemble becomes smaller and finally disappears for increasing length of the light period. It is also possible to calculate the amplitude and width of the narrow peak as a function of $T_{0}$. Since the resulting expressions are quite complex we do not give them here explicitly.

It may seem surprising at first glance that the narrow peak in the resonance-fluorescence spectrum disappears when one observes the spectrum in a light period instead of looking at the complete ensemble. However, there is a fairly simple explanation for this behavior. In a recent publication [9] we have shown that the narrow peak in the spectrum of resonance fluorescence can be understood if one models the resonance fluorescence of the $V$ system by a radiating two-level system whose resonance fluorescence is randomly turned on and off. The process of turning the resonance fluorescence on and off is described by a random telegraph process with Poissonian distributions for the jump times from 0 to 1 and vice versa. This random modulation has the effect that the Rayleigh peak, which describes the coherent part of the radiation scattered by the two-level system, splits into a $\delta$ function and a narrow Lorentzian. The latter gives rise to the narrow peak in the spectrum of resonance fluorescence. This model gives good quantitative agreement with the analytical results derived from Bloch equations and the quantum re- 


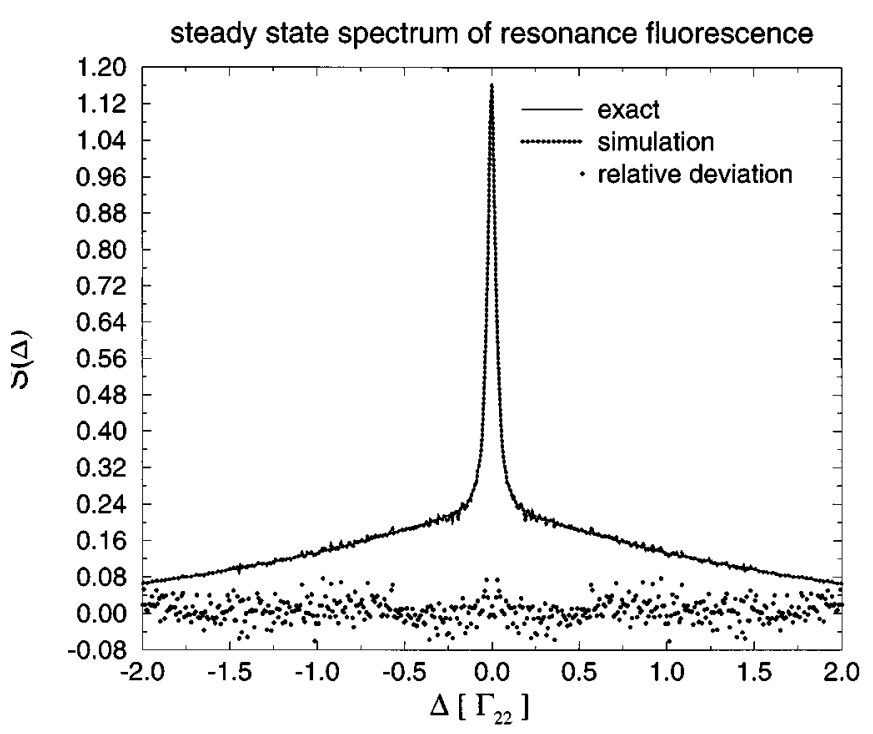

FIG. 4. Comparison of the exact steady-state spectrum of resonance fluorescence (solid line) with the results of a simulation with 1000 runs (dotted line). The parameters are the same as in Fig. 3. The relative deviation of the simulation results from the exact results is indicated by dots.

gression theorem. If we observe the spectrum of resonance fluorescence in a light period the resonance fluorescence of the strong transition is no longer modulated by dark periods, which in turn has the effect that the Rayleigh peak is no longer broadened. Since the narrow peak in the spectrum of resonance fluorescence is just the broadened part of the Rayleigh peak we understand that it must be missing when observed in a light period, because there is no modulation of resonance fluorescence due to dark periods.

\section{DISCUSSION OF RESULTS. CONCLUSIONS}

This paper was motivated by questions about spectral properties of a single, laser-driven multilevel atom, as op-

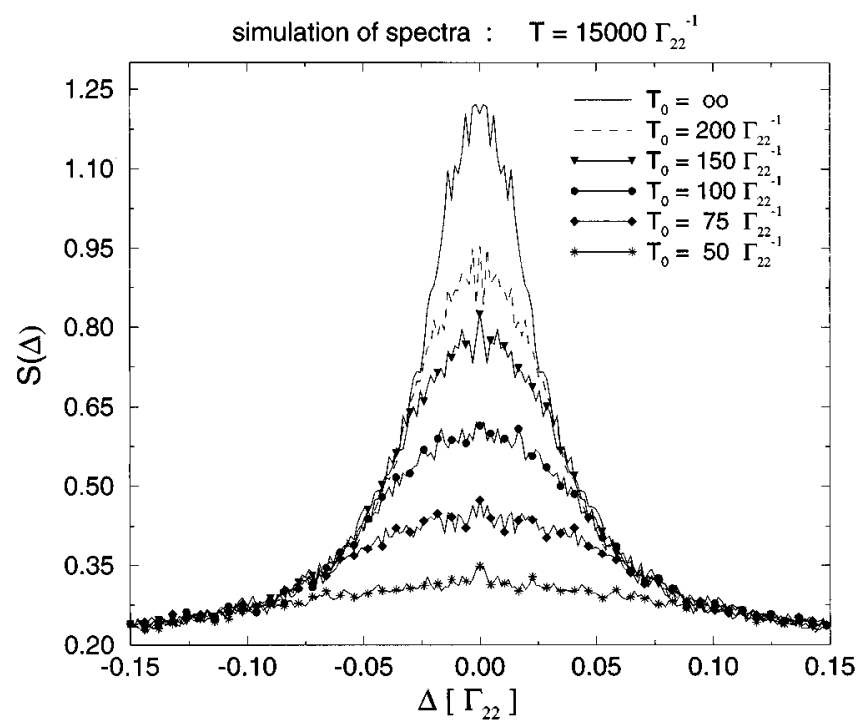

FIG. 5. Simulation of the spectrum of resonance fluorescence in a light period of length $T=15000 \Gamma_{22}^{-1}$ for different values of $T_{0}$ and the parameters $\Omega_{2}=2 \Gamma_{22}, \Omega_{3}=0.2 \Gamma_{22}$, and $\Delta_{2}=\Delta_{3}=0$. With decreasing $T_{0}$ the central peak decreases and finally disappears.

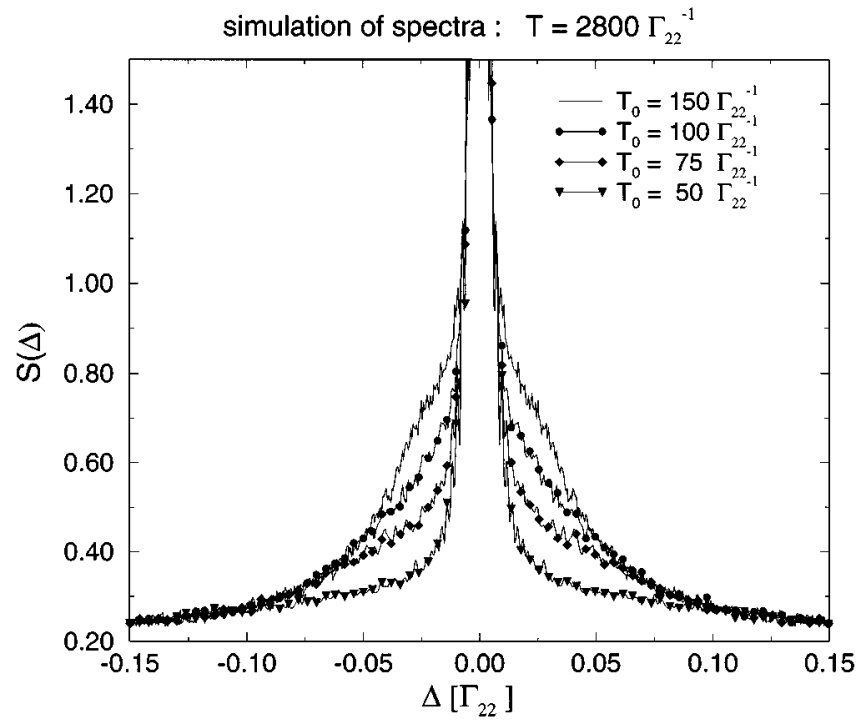

FIG. 6. Simulation of the spectrum of resonance fluorescence for the same parameters as in Fig. 5, but now for light periods with lengths longer than $T=2800 \Gamma_{22}^{-1}$. Because of the shorter light periods as compared to Fig. 5 the Rayleigh peak is much more broadened. Again the amplitude of the narrow peak decreases with $T_{0}$.

posed to questions about spectral properties of a complete ensemble. As is known from a previous paper of ours [9], unexpected spectral features can occur in the steady-state ensemble spectrum of systems which exhibit electron shelving, i.e., macroscopic light and dark periods. It therefore suggests itself to study the spectrum of radiation emitted in a light period. This is a question which can only be studied for single systems since light and dark periods of different atoms in general overlap. In order to know that a driven atom is in a light period, the emitted photons have to be monitored in a time-resolved way. To circumvent any problems with the time-energy uncertainty relation we have developed a theory

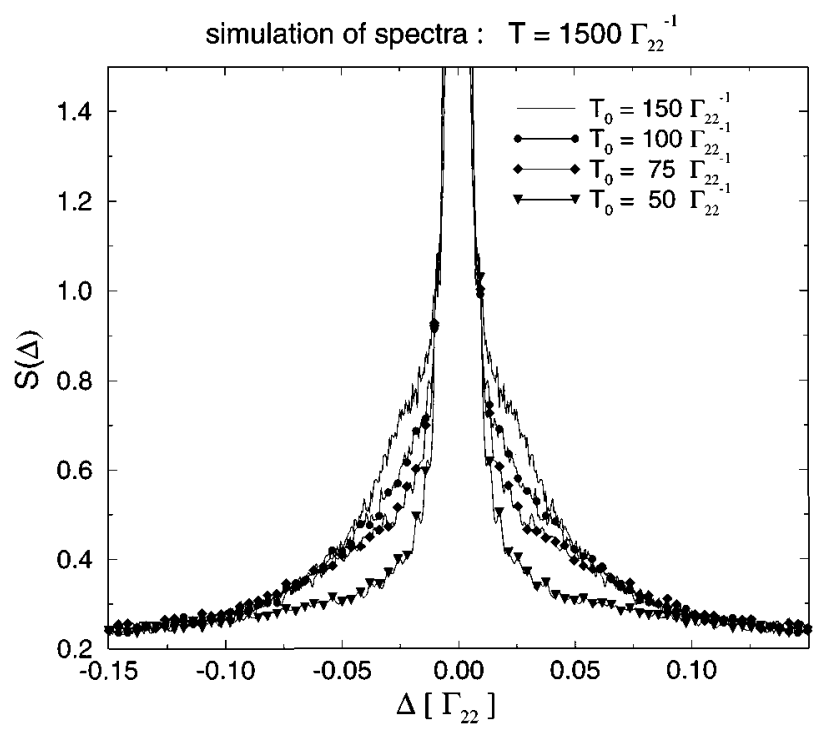

FIG. 7. Same simulation as Fig. 6, but now for light periods longer than $T=1500 \Gamma_{22}^{-1}$. The Rayleigh peak is even more broadened than in Fig. 6. The amplitude of the narrow peak decreases with $T_{0}$. 


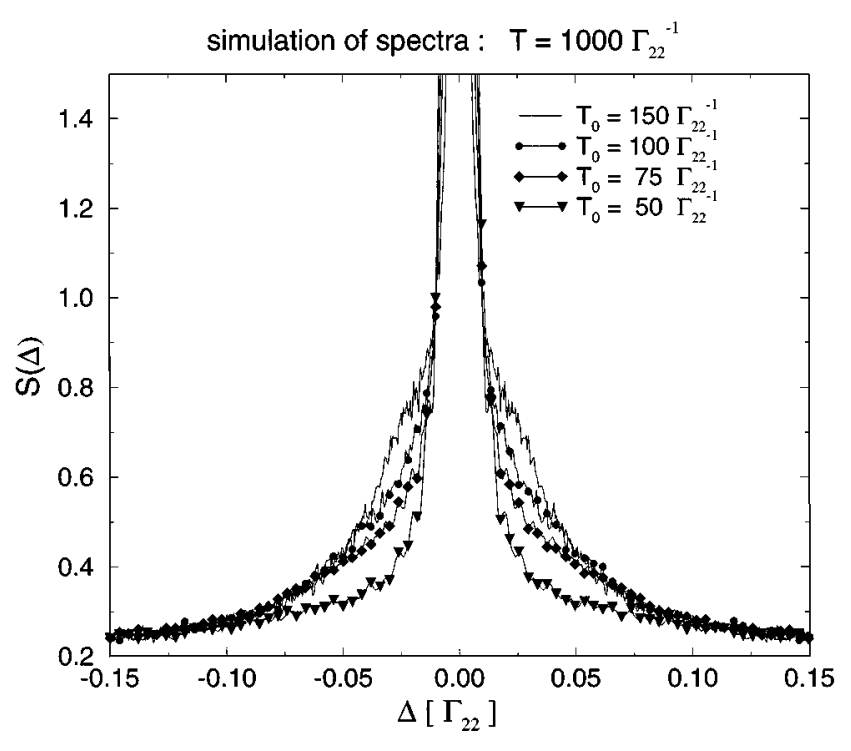

FIG. 8. Same simulation as Figs. 6 and 7, but now for light periods longer than $T=1000 \Gamma_{22}^{-1}$.

which allows us to simultaneously study time-resolved properties, i.e., photon statistics, in one solid angle $\Omega_{B}$ and spectral properties in another, $\Omega_{S}$.

This led us to the notion of the conditional spectrum, for example, the spectrum of the radiation emitted into $\Omega_{S}$ until some time $t$, under the condition that a particular temporal emission pattern of photons is found in $\Omega_{B}$. The theory developed above allows one to calculate, for given emission pattern, the expectation value of $a_{\mathbf{k} \lambda}^{\dagger} a_{\mathbf{k} \lambda}$ and, more generally, the expectation value of $\left(a_{\mathbf{k} \lambda}^{\dagger}\right)^{m}\left(a_{\mathbf{k} \lambda}\right)^{n}$. Physically this corresponds to a situation where one considers only atoms with the same temporal pattern of emission into the solid angle $\Omega_{B}$ between time 0 and $t$, measures the spectrum in the solid angle $\Omega_{S}$ for each atom, and forms the mean of these spectra. In general there will be deviations of the individual spectra from the mean, i.e., fluctuations. These can also be determined by the theory and they will decrease with increasing radiation analyzed by the spectrometer, and thus with time $t$ and the aperture.
The theory has been applied to a single driven three-level $V$ system and to spectral properties of radiation emitted into a solid angle $\Omega_{S}$ during light periods, with the light periods monitored in a solid angle $\Omega_{B}$. The photon statistics of an atom with light and dark periods have two very different time scales, and one usually chooses an intermediate time $T_{0}$ to characterize a light period as an emission pattern with $T_{0}$ as maximal waiting time between photons emitted into $\Omega_{B}$. We have slightly generalized this by allowing arbitrarily large waiting times. This has permitted us to study the transition to the stationary spectrum of the complete ensemble.

A collection of $V$ systems was considered, all in a light period starting at time 0 and lasting at least until time $t$. All temporal emission patterns in the solid angle $\Omega_{B}$ until time $t$, compatible with such light periods, were considered. For each pattern the corresponding conditional spectrum in $\Omega_{S}$ at time $t$ was calculated and then the mean of the spectra over all emission patterns was formed.

To a single $V$ system this applies as follows. One samples the light periods of a single atom and determines, for each of the sampled light periods with the respective temporal emission pattern in the solid angle $\Omega_{B}$, the spectrum of the radiation emitted into the solid angle $\Omega_{S}$ from the start of the period until $t$ sec later. The mean of these spectra is precisely what we have calculated and what we have called the spectrum of an atom in a light period. This should be amenable to experimental verification for a single ion in a trap.

We have presented numerical calculations of spectra in a light period for a $V$ system which has one metastable state and which is driven by two lasers. In a previous paper [9] we had studied the stationary ensemble spectrum of such systems and had found, for the above parameter range, an additional, extremely narrow peak in the spectrum. We had explained this peak through the modulation of the radiation by the dark periods. If correct, this explanation would imply that the peak should disappear for the spectrum in a light period. This is indeed what we found.

We thus conclude that the spectrum in a light period of a single atom may be significantly different from the ensemble spectrum. It would be interesting to check this predicted effect experimentally.
[1] B. R. Mollow, Phys. Rev. 188, 1969 (1969); Phys. Rev. A 5, 1522 (1972); 5, 2217 (1972).

[2] R. E. Grove, F. Y. Wu, and S. Ezekiel, Phys. Rev. Lett. 35, 1426 (1975); W. Hartig, W. Rasmussen, R. Schieder, and H. Walther, Z. Phys. A 278, 205 (1976).

[3] H. J. Carmichael and D. F. Walls, J. Phys. B 9, L43 (1976); 9, 1199 (1976).

[4] H. J. Kimble, M. Dagenais, and L. Mandel, Phys. Rev. Lett. 39, 691 (1977); Phys. Rev. A 18, 201 (1978); F. M. Rateike, G. Leuchs, and H. Walther, in Dissipative Systems in Quantum Optics, edited by R. Bonifacio (Springer, Berlin, 1982).

[5] F. Diedrich and H. Walther, Phys. Rev. Lett. 58, 203 (1987).

[6] H. G. Dehmelt, Bull. Am. Phys. Soc. 20, 60 (1975); R. J. Cook and H. J. Kimble, Phys. Rev. Lett. 54, 1023 (1985); C. CohenTannoudji and J. Dalibard, Europhys. Lett. 1, 441 (1986); A.
Schenzle, R. G. DeVoe, and R. G. Brewer, Phys. Rev. A 33, 2127 (1986); J. Javanainen, ibid. 33, 2121 (1986); D. T. Pegg, R. Loudon, and P. L. Knight, ibid. 33, 4085 (1986); G. Nienhuis, ibid. 35, 4639 (1987); P. Zoller, M. Marte, and D. J. Walls, ibid. 35, 198 (1987); S. Reynaud, J. Dalibard, and C. Cohen-Tannoudji, IEEE J. Quantum Electron. 24, 1395 (1988); M. Porrati and S. Putterman, Phys. Rev. A 36, 925 (1987); 39, 3010 (1989); M. S. Kim and P. L. Knight, ibid. 40, 215 (1989).

[7] J. C. Bergquist, R. G. Hulet, W. M. Itano, and D. J. Wineland, Phys. Rev. Lett. 57, 1699 (1986); Th. Sauter, R. Blatt, W. Neuhauser, and P. E. Toschek, Opt. Commun. 60, 287 (1986); W. Nagourney, J. Sandberg, and H. Dehmelt, Phys. Rev. Lett. 56, 2797 (1986); J. C. Bergquist, W. M. Itano, R. G. Hulet, and D. J. Wineland, Phys. Scr. T22, 79 (1988). 
[8] The spectrum of a single ion was measured recently for the first time in Hamburg by Toschek and co-workers; P. Toschek (private communication).

[9] G. C. Hegerfeldt and M. B. Plenio, Phys. Rev. A (to be published). Cf. also B. M. Garraway, M. S. Kim, and P. L. Knight, Opt. Commun. (to be published).

[10] C. Cohen-Tannoudji and J. Dalibard, Europhys. Lett. 1, 441 (1986); P. Zoller, M. Marte, and D. J. Walls, Phys. Rev. A 35, 198 (1987); M. Porrati and S. Puttermann, ibid. 39, 3010 (1989); M. S. Kim and P. L. Knight, ibid. 40, 215 (1989); T. S. Wilser, Doctoral thesis, University of Göttingen, 1991; G. C. Hegerfeldt and T. S. Wilser, in Proceedings of the II. International Wigner Symposium, Goslar 1991, edited by H. D. Doebner, W. Scherer, and F. Schroeck (World Scientific, Singapore, 1992); J. Dalibard, Y. Castin, and K. Mølmer, Phys. Rev. Lett. 68, 580 (1992); K. Mølmer, Y. Castin, and J. Dalibard, J. Opt. Soc. Am. B 10, 524 (1993); H. J. Carmichael, in An Open Systems Approach to Quantum Optics, Lecture Notes in Physics Vol. m18 (Springer, Berlin, 1993).

[11] G. C. Hegerfeldt, Phys. Rev. A 47, 449 (1993).

[12] C. W. Gardiner, A. S. Parkins, and P. Zoller, Phys. Rev. A 46, 4363 (1992); R. Dum, A. S. Parkins, P. Zoller, and C. W. Gardiner, ibid. 46, 4382 (1992).

[13] K. Mølmer, Y. Castin, and J. Dalibard, J. Opt. Soc. Am. B 10, 524 (1993).

[14] Usually the spectrum of resonance fluorescence is obtained from the Fourier transform of the $g^{(1)}$ function or, equivalently, of the atomic correlation function $\left\langle\sigma_{21}(t+\tau) \sigma_{12}(t)\right\rangle$. However, a simulation procedure based on these correlation functions cannot be interpreted in a straightforward way for a single system. We therefore use the photon number operator. For the stationary spectrum of the complete ensemble both definitions are equivalent, cf. G. S. Agarwal, Quantum Optics: Quantum Statistical Theories of Spontaneous Emission and Their Relation to Other Approaches, Springer Tracts in Modern Physics Vol. 70 (Springer-Verlag, Berlin, 1974).

[15] Cf. G. C. Hegerfeldt and T. S. Wilser, in Ref. [10], and Ref. [11].

[16] M. B. Plenio, Doctoral thesis, University of Göttingen, 1994.

[17] G. Lüders, Ann. Phys. (Leipzig) 8, 323 (1951); J. von Neumann, Mathematical Foundations of Quantum Mechanics (Princeton University Press, Princeton, NJ, 1955); Chap. V.1. For observables with degenerate eigenvalues von Neumann's prescription differs from the current form of the projection postulate as formulated by Lüders.

[18] For irradiation with lasers this is the case, but not for irradiation with incoherent, broadband, light, cf. G. C. Hegerfeldt and M. B. Plenio, Phys. Rev. A 47, 2186 (1993).

[19] For convenience we will neglect the coupling of the metastable state to the quantized radiation field. The introduction of a small decay rate for this level does not change the results in any essential way. This is discussed by us in more detail in Ref. [9].

[20] C. Cohen-Tannoudji, J. Dupont-Roc, and G. Grynberg, AtomPhoton Interactions (John Wiley \& Sons, New York, 1992).

[21] M. D. Srinivas and E. B. Davies, Opt. Acta 28, 981 (1981).

[22] Cf. the last two articles of Ref. [1].

[23] The sidebands occurring for $\Omega_{2}>\Gamma_{22}$ are still too close to the central peak; cf. H. J. Carmichael, Ref. [10], p. 51. 